\title{
Molecular emission near metal interfaces: the polaritonic regime
}

\author{
Joel Yuen-Zhou ${ }^{1}$, Semion K. Saikin ${ }^{2}$, Vinod M. Menon ${ }^{3}$ \\ ${ }^{1}$ Department of Chemistry and Biochemistry, University of California San Diego, La Jolla, CA, USA ${ }^{*}$ \\ ${ }^{2}$ Department of Chemistry and Chemical Biology, Harvard University, Cambridge, MA, USA. \\ ${ }^{2}$ Institute of Physics, Kazan Federal University, Kazan, Russian Federation. and \\ ${ }^{4}$ Department of Physics, Graduate Center and City College of New York, \\ City University of New York, New York, New York, USA.
}

\begin{abstract}
The strong coupling of a dense layer of molecular excitons with surface-plasmon modes in a metal gives rise to polaritons (hybrid light-matter states) called plexcitons. Surface plasmons cannot directly emit into (or be excited by) free-space photons due to the fact that energy and momentum conservation cannot be simultaneously satisfied in photoluminescence. Most plexcitons are also formally non-emissive, even though they can radiate via molecules upon localization due to disorder and decoherence. However, a fraction of them are bright even in the presence of such deleterious processes. In this letter, we theoretically discuss the superradiant emission properties of these bright plexcitons, which belong to the upper energy branch and reveal huge photoluminescence enhancements compared to bare excitons. Our study generalizes the well-known problem of molecular emission next to a metal interface to collective molecular states and provides new design principles for the control of photophysical properties of molecular aggregates using polaritonic strategies.
\end{abstract}

The study of molecular photoluminescence (PL) next to a metal-dielectric interface dates back to a classic experiment reported by Drexhage, Kuhn, and coworkers almost fifty years ago [1, 2]. By controlling the distance between a molecular emitter and a metal-mirror film, the aforementioned authors showed that the observed PL rate oscillated and then monotonically increased for short distances. These oscillations were attributed to interferences between the free-space and reflected light from the mirror, and the monotonic decay was associated to irreversible energy transfer to surface plasmons (SPs) in the metal. These observations were later fully elucidated in a theory provided by Chance, Prock, and Silbey (CPS) 3, 4, by adapting the results of an even older problem of antenna radiation next to the surface of the Earth, whose mathematical solution was offered by Sommerfeld as early as 1909 [5]. This problem has been revisited countless times to understand molecular energy transfer processes in condensed phases.

In this letter, we study a variation of the aforementioned problem which offers new phenomenology that, as far as we are aware, has not been reported before. We study PL originating from (delocalized) molecular excited states (excitons) when they are strongly coupled to a SP metal film (see Fig. 1). We consider a layer of $N_{x} N_{y} N_{z}$ molecules (of thickness $W_{z}$ ) placed on top of a thin dielectric spacer (of thickness $z_{0}$ ), which is on a thick metal film. When the molecular layer is sufficiently dense, the energy transfer between the exciton and SP modes is reversible and faster than each of the decay rates [6, 7]. The resulting eigenmodes are no longer purely plasmonic or excitonic, but rather, polaritonic, being coherent superpositions of such modes. Polaritons arising from SPs and excitons are termed plexcitons [8]12. As

\footnotetext{
* joelyuen@ucsd.edu
}

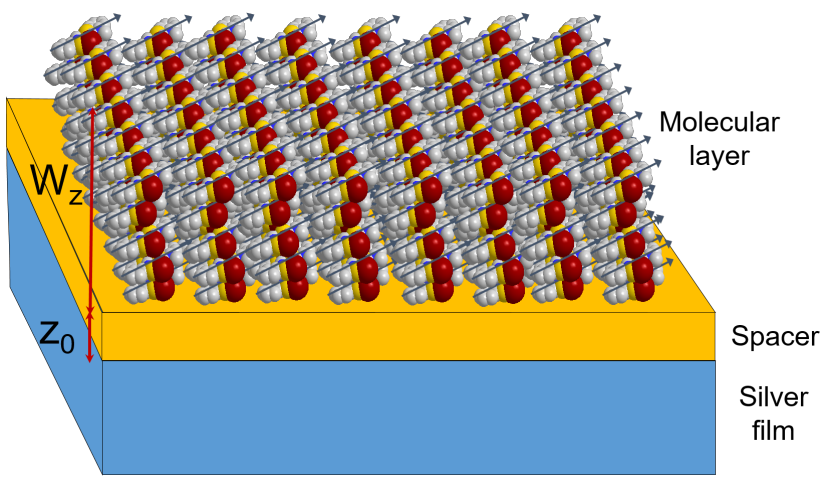

Figura 1. Plexciton setup. A molecular layer of thickness $W_{z}$ sits on top of a thin dielectric spacer of thickness $z_{0}$, which in turn, is placed on a metal film. Strong coupling between excitons in the molecular layer and surface-plasmons (SPs) in the metal film imply energy exchange between the excitons and SPs is much faster than their respective decay processes, giving rise to polaritonic excitations called plexcitons.

with any polaritonic system, the transmission spectrum of the plexciton system at the wavevector giving rise to resonance between the uncoupled exciton and SP bands shows a Rabi splitting (anticrossing) between two new bands, named upper and lower plexcitons (UPs, LPs), according to their energy ordering [7. Molecular polaritons have recently been the subject of intense investigation, as they offer new ways for coherent control of molecular processes [13] such as changes on reaction rates and thermodynamics by "imprinting" electromagnetic coherence directly onto the molecular states [14-20]. They also provide new platforms to induce remote energy transfer [21 23] and to recreate exotic topological [12, 24] and many-body phenomena at room temperature in tabletop experiments [25-27]. In this study, we highlight superradiant properties [28, 29] that plexcitons exhibit which are not encountered in the weak coupling regime 
of a bare molecule next to a metal surface. These properties can potentially be harnessed for light harvesting and energy routing purposes in molecular materials and device applications.

We first briefly lay out a quantum-mechanical formalism to describe the plexciton system (see details in Supporting Information, SI-I, II). Writing the Hamiltonian as $H=\sum_{k} H_{k}$, where the sum runs over a discrete set of in-plane exciton wavevectors $\boldsymbol{k}$, we have [6, 7]

$$
\begin{aligned}
H_{\boldsymbol{k}} & =\hbar \omega_{\boldsymbol{k}}^{S P} a_{\boldsymbol{k}}^{\dagger} a_{\boldsymbol{k}}+\hbar \omega_{e} \sigma_{\boldsymbol{k}}^{\dagger} \sigma_{\boldsymbol{k}} \\
& +\left(\mathcal{J}_{\boldsymbol{k}} \sigma_{\boldsymbol{k}}^{\dagger} a_{\boldsymbol{k}}+\text { h.c. }\right)+H_{\text {dark, } \boldsymbol{k}}+H_{\text {umklapp }, \boldsymbol{k}}
\end{aligned}
$$

Here, $\hbar \omega_{\boldsymbol{k}}^{S P}$ and $a_{\boldsymbol{k}}^{\dagger}\left(a_{\boldsymbol{k}}\right)\left[\hbar \omega_{e}\right.$ and $\left.\sigma_{\boldsymbol{k}}^{\dagger}\left(\sigma_{\boldsymbol{k}}\right)\right]$ are the energy and creation (annhilation) operator of a $\boldsymbol{k}$ th SP [exciton]; $\mathcal{J}_{\boldsymbol{k}}$ is the collective SP-exciton coupling. $H_{\text {dark, } \boldsymbol{k}}$ describes dark excitons at the bare exciton energy $\hbar \omega_{e}$, which do not couple directly to SPs [20]; we disregard $H_{\text {umklapp, }}$ as a negligible off-resonant contribution due to coupling with high-wavevector SP modes. Ignoring also $H_{d a r k, \boldsymbol{k}}$ for the time-being (we will discuss it at the end of the letter), $H_{\boldsymbol{k}}$ in Eq. (1) becomes a two-level system for every $\boldsymbol{k}$, and can be diagonalized to yield two plexciton (polariton) states of the form $\left|y_{\boldsymbol{k}}\right\rangle=\left[\zeta_{y_{\boldsymbol{k}}}^{(S P)} a_{\boldsymbol{k}}^{\dagger}+\zeta_{y_{\boldsymbol{k}}}^{(e x c)} \sigma_{\boldsymbol{k}}^{\dagger}\right]|\mathrm{vac}\rangle$ with eigenenergies $\hbar \omega_{y_{k}}$, where $y= \pm$ are labels for the UP (LP), and $|\mathrm{vac}\rangle=\left|g ; 0_{S P} ; 0_{U H P}\right\rangle$ is the tensor product of the ground state for the molecular degrees of freedom $(|g\rangle)$ and the vacuum for the SP modes $\left(\left|0_{S P}\right\rangle\right)$; see Fig. 2 a.

Fig. 2 a shows a dispersion energy plot for exciton, $\mathrm{SP}$, and resulting plexciton bands assuming $\omega_{e}=3 \mathrm{eV}$, $\left|\boldsymbol{\mu}_{e g}\right|=10$ Debye, and relative permittivities $\epsilon_{d}=1,3$ and $\epsilon_{m}(\omega)=3,7-\frac{(8,6 \mathrm{eV})^{2}}{\omega^{2}}$ for the molecular (dielectric) layer and metal film, respectively. We used $\rho=$ $10^{9}$ molecules $/ \mu \mathrm{m}^{-3}, z_{0}=1 \mathrm{~nm}$, and $W_{z}=200 \mathrm{~nm}$, to account for representative parameters in the literature 6]. We took isotropic averages of $\mathcal{J}_{\boldsymbol{k}}$ to describe a molecular layer with orientational disorder. Conservation of energy and in-plane momentum constrains the allowed PL processes: an excitation of frequency $\omega$ and in-plane wavevector $\boldsymbol{k}$ must output a propagating photon with the same properties. This means that, for PL to occur, there needs to be a real-valued $k_{z d}$ satisfying

$$
\omega=\frac{c}{\sqrt{\epsilon_{d}}} \sqrt{|\boldsymbol{k}|^{2}+\left|k_{z d}\right|^{2}} .
$$

It is well known this is not a possibility for SP modes [30, whose dispersion is to the right of the "light-line," $\omega_{\boldsymbol{k}}^{S P}<$ $\omega_{\boldsymbol{k}}^{l l} \equiv \frac{c}{\sqrt{\epsilon_{d}}}|\boldsymbol{k}|$, and thus, must be probed by coupling to gratings 31 or nanoparticles 32, for example. The same holds formally true for LPs $\left(\omega_{-_{k}}<\omega_{k}^{l l}\right)$ and for a subset of UPs; to distinguish them from the dark exciton states (eigenstates of $H_{d a r k, k}$ ), we shall call them non-emissive states. Importantly, there is another subset of UPs that are to the left of the light-line, thus being formally bright
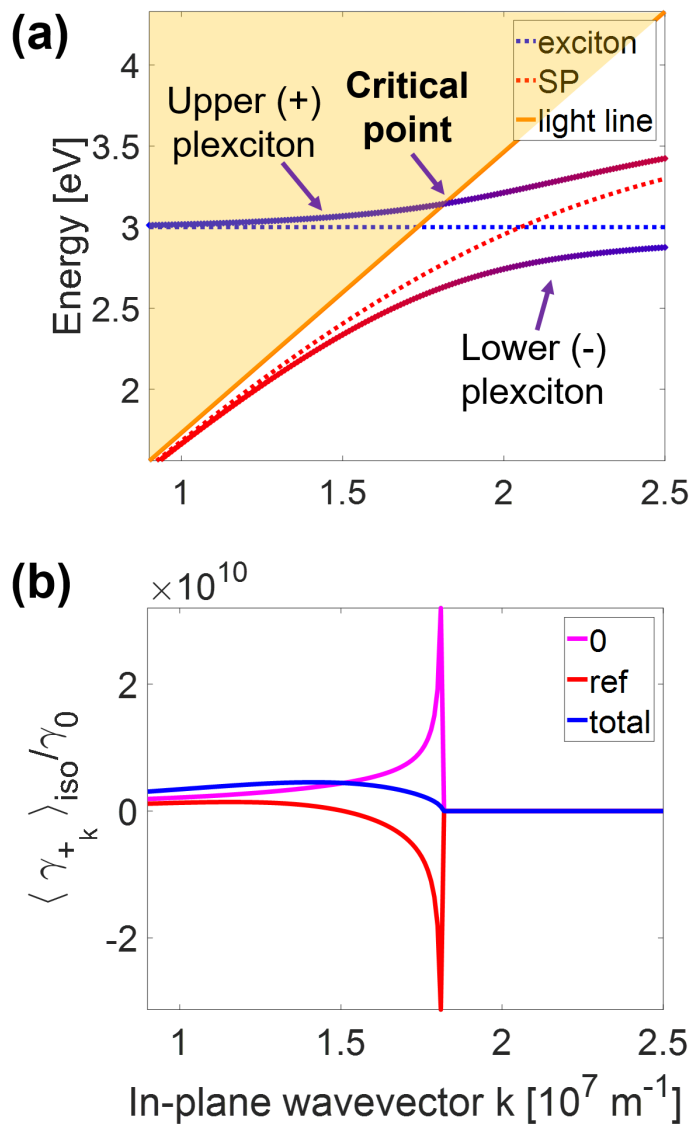

Figura 2. (a) Dispersion plot of excitations for an isotropic molecular layer. When the (flat) exciton (dashed blue) and the SP (dashed red) bands couple, they give rise to upper and lower plexciton (UP, LP). The UP $\left|+_{\boldsymbol{k}}\right\rangle$ interpolates between exciton and SP between $|\boldsymbol{k}| \approx 0$ and $|\boldsymbol{k}| \rightarrow \infty$; the converse is true for the LP $(|-\boldsymbol{k}\rangle)$. Strong hybridization occurs in the anticrossing region. The yellow-shaded region contains states that emit photons, while the right region contains states that cannot due to mismatch in energy and in-plane wavevector with respect to free-space photons. The critical point at $\boldsymbol{k}=$ $\boldsymbol{k}^{*}$ separates bright and non-emissive UPs. (b) PL rates for the $U P$ band $\left\langle\gamma_{+_{k}}\right\rangle_{\text {iso }}=\sum_{i}\left\langle\gamma_{+_{k}, i}\right\rangle_{\text {iso }}$ normalized to the singlemolecule rate $\gamma_{S M}$. Contributions to $\left\langle\gamma_{+_{\boldsymbol{k}}}\right\rangle_{\text {iso }}$ correspond to direct $(i=0)$, and interference terms between free-space and reflected waves $(i=r e f)$. The divergences in $\left\langle\gamma_{+_{\boldsymbol{k}^{*}, i}}\right\rangle_{\text {iso }}$ are van Hove singularities.

and featuring PL (see Fig. 2a). We then ask: what is the rate of PL from these UPs, and how does the brightto-non-emissive transition occur as a function of $\boldsymbol{k}$ ? To answer these questions, we compute the PL rate of a state $\left|y_{\boldsymbol{k}}\right\rangle$ into the free-space radiative modes of the upper-halfplane (UHP) of the plexciton setup. The corresponding Wigner-Weiskopf expression (Fermi golden rule) is 33 , 34 
$\gamma_{y_{\boldsymbol{k}}}=\frac{2 \pi}{\hbar} \sum_{\boldsymbol{K}, \chi}\left|\left\langle\operatorname{vac} ;(\boldsymbol{K}, \chi)_{U H P}\left|H_{i n t}\right| y_{\boldsymbol{k}} ; 0_{U H P}\right\rangle\right|^{2} \delta\left(\hbar \omega_{y_{\boldsymbol{k}}}-\hbar \omega^{\boldsymbol{s}}\right.$

where $H_{\text {int }}=-\sum_{\boldsymbol{n} s} \hat{\boldsymbol{\mu}}_{\boldsymbol{n} s} \cdot \hat{\mathcal{E}}_{U H P}\left(\boldsymbol{r}_{\boldsymbol{n} s}\right)$ contains the interaction between each of the molecules in the slab and the electric field at the UHP. In Eq. (3), $\left|0_{U H P}\right\rangle$ denotes the UHP photonic vacuum state. The matrix element couples an initial state with a plexciton and no UHP photons $\left|y_{\boldsymbol{k}} ; 0_{U H P}\right\rangle$ with a final state featuring no plexcitons and an UHP photon with energy $\hbar \omega_{\boldsymbol{K}}^{U H P}=\frac{\hbar c|\boldsymbol{K}|}{\sqrt{\epsilon_{d}}},\left|\operatorname{vac} ;(\boldsymbol{K}, \chi)_{U H P}\right\rangle=b_{\boldsymbol{K}, \chi}^{\dagger}\left|\operatorname{vac} ; 0_{U H P}\right\rangle$, where $b_{\boldsymbol{K}, \chi}^{\dagger}\left(b_{\boldsymbol{K}, \chi}\right)$ is the creation (annhilation) operator of photons at the UHP mode with (three-dimensional) wavevector $\boldsymbol{K}=\left(K_{x}, K_{y}, K_{z d}\right)$ and polarization in$\operatorname{dex} \chi=s, p$, which satisfies the commutation relation $\left[b_{\boldsymbol{K}, \chi}, b_{\boldsymbol{K}^{\prime}, \chi^{\prime}}^{\dagger}\right]=\delta_{\boldsymbol{K}, \boldsymbol{K}^{\prime}} \delta_{\chi, \chi^{\prime}}$. The electric field at the UHP is given by a collection of radiative modes. Using the modal representation given by Arnoldus and George [35],

$$
\begin{aligned}
\hat{\mathcal{E}}_{U H P}(\boldsymbol{r}) & =\sum_{\boldsymbol{K}, \chi} \Theta(-\boldsymbol{K} \cdot \hat{\boldsymbol{z}})\left\{\frac{\left(b_{\boldsymbol{K}, \chi}+b_{\tilde{\boldsymbol{K}}, \chi}\right)}{\sqrt{1+\left|r_{\boldsymbol{K} \chi}\right|^{2}}} \sqrt{\frac{\hbar \omega_{\boldsymbol{K}}^{U H P}}{2 \epsilon_{0} \epsilon_{d} V}}\right. \\
& \left.\times\left[\boldsymbol{e}_{\boldsymbol{K}, \chi} e^{i \boldsymbol{K} \cdot \boldsymbol{r}}+r_{\boldsymbol{K}, \chi} e^{i \tilde{\boldsymbol{K}} \cdot \boldsymbol{r}} \boldsymbol{e}_{\tilde{\boldsymbol{K}}, \chi}\right]+\text { h.c. }\right\} .
\end{aligned}
$$

The radiative modes can have $s$ or $p$ polarization, $\boldsymbol{e}_{\boldsymbol{K}, s}=\frac{\boldsymbol{K} \times \hat{\boldsymbol{z}}}{|\boldsymbol{K} \times \hat{\boldsymbol{z}}|}=\frac{K_{y} \hat{\boldsymbol{x}}-K_{x} \hat{\boldsymbol{y}}}{\sqrt{K_{x}^{2}+K_{y}^{2}}}$ and $\boldsymbol{e}_{\boldsymbol{K}, p}=\frac{\boldsymbol{e}_{\boldsymbol{K} s} \times \boldsymbol{K}}{\left|\boldsymbol{e}_{\boldsymbol{K}} \times \boldsymbol{K}\right|}=$ $\frac{-K_{z d}\left(K_{x} \hat{\boldsymbol{x}}+K_{y} \hat{\boldsymbol{y}}\right)}{|\boldsymbol{K}| \sqrt{K_{x}^{2}+K_{y}^{2}}}+\frac{\sqrt{K_{x}^{2}+K_{y}^{2}} \hat{z}}{|\boldsymbol{K}|} \cdot V$ is the quantization volume of the UHP, $\Theta$ is the Heavyside step function, and $\tilde{\boldsymbol{K}}=\boldsymbol{K}-2 \boldsymbol{K} \cdot \hat{\boldsymbol{z}} \hat{\boldsymbol{z}}$ is the reflected wavevector for an incident wave into the metal with $K_{z d}<0$ and Fresnel coefficients $r_{\boldsymbol{K}, s}=\frac{K_{z d}-K_{z m}}{K_{z d}+K_{z m}}$ and $r_{\boldsymbol{K}, p}=\frac{\epsilon_{m} K_{z d}-\epsilon_{d} K_{z m}}{\epsilon_{m} K_{z d}+\epsilon_{d} K_{z m}}$ [31, where $K_{z m}(\boldsymbol{K}, \omega)=-\sqrt{\epsilon_{m}(\omega) \frac{\omega^{2}}{c^{2}}-\left|\boldsymbol{K}_{\perp}\right|^{2}}$. Note that although $K_{z d} \in \Re, K_{z m} \in \Im$ given that the UP frequency is typically below the asymptotic $k \rightarrow \infty$ SP frequency, $\omega_{\boldsymbol{K}}^{U H P}<\frac{\omega_{P}}{\sqrt{\epsilon_{d}+\epsilon_{\infty}}}$. This in turn yields $\left|r_{\boldsymbol{K} \chi}\right|^{2}=1$, i.e., lossless metals are perfectly reflective 35 .

Eq. (4) implies the rate $\gamma_{y_{k}}$ calculated through Eq. (3) can be partitioned into two contributions, $\gamma_{y_{k}}=$ $\gamma_{y_{k}, 0}+\gamma_{y_{k}, r e f}$, corresponding to the direct term associated with the free-space and reflected electric fields independently, and the interference term between them, as done with single molecule calculations [4, 36, 37]. We present results normalized to $\gamma_{S M, 0}=\frac{\sqrt{\epsilon_{d}}}{3 \pi \epsilon_{0} \hbar}\left(\frac{\omega_{e}}{c}\right)^{3}\left|\boldsymbol{\mu}_{e g}\right|^{2}$, the single-molecule PL rate in free-space, which attains a value of $0,5 \mathrm{~ns}^{-1}$ for the present parameters. Recall that the single-molecule PL rate scales as $\omega_{e}^{3}$ because it can radiate across all solid angles (the number of accesible radiative modes grows as $\omega_{e}^{2}$ ) and it is proportional to the intensity of the electric field $\left(\propto \omega_{e}\right)$ [33, 34]. The evaluation of Eq. (3) is presented in SI-III, where we also whing in great detail an alternative route to obtain the same results using the dyadic Green's function formalism [31, 38, 39]. Here, we limit ourselves to discuss the isotropically distributed molecular layer, which already captures the essential features of the general solution; denoting isotropic averages by $\langle\cdot\rangle_{i s o}$, we obtain (see Fig. $2 \mathrm{~b})$,

$$
\begin{aligned}
\frac{\left\langle\gamma_{y_{\boldsymbol{k}}, i}\right\rangle_{i s o}}{\gamma_{S M, 0}} & =\left(\frac{2 \pi c}{\sqrt{\epsilon_{1}}}\right)\left|\zeta_{y_{\boldsymbol{k}}}^{(e x c)}\right|^{2} \Theta\left(\omega_{y_{\boldsymbol{k}}}-\frac{c}{\sqrt{\epsilon_{d}}}|\boldsymbol{k}|\right) \rho \\
& \times\left(\frac{\omega_{y_{\boldsymbol{k}}}^{2} \frac{1}{k_{z d}}}{\omega_{e}^{3}}\right) \frac{f_{i}}{\int_{z_{0}}^{z_{f}} d z\left|J_{\boldsymbol{k}}(z)\right|^{2}}
\end{aligned}
$$

where

$$
\begin{aligned}
f_{0} & =\left|\int_{z_{0}}^{z_{f}} d z J_{\boldsymbol{k}}(z) e^{-i k_{z d} z}\right|^{2}, \\
f_{\text {ref }} & =\frac{1}{2} \Re\left\{\left(r_{\boldsymbol{k}-k_{z d} \hat{\boldsymbol{z}}, s}-r_{\boldsymbol{k}-k_{z d} \hat{\boldsymbol{z}}, p} \frac{k_{d z}^{2}-|\boldsymbol{k}|^{2}}{k_{d z}^{2}+|\boldsymbol{k}|^{2}}\right)\right. \\
& \left.\times\left[\int_{z_{0}}^{z_{f}} d z J_{\boldsymbol{k}}(z) e^{i k_{z d} z}\right]^{2}\right\} .
\end{aligned}
$$

Eqs. (5) and (6) are the main results of this letter. To capture the essential physics behind the PL trends, we have so far considered a lossless metal. However, as we demonstrate in SI-IV] the formalism of macroscopic quantum electrodynamics (M-QED) 40,42 validates these expressions even when the metal is lossy, where $\left|r_{\boldsymbol{k}-k_{z d} \hat{\boldsymbol{z}}, \chi}\right|<1$. Let us provide a physical interpretation of these results. The PL rate for a plexciton state is proportional to its exciton population $\left|\zeta_{y_{k}}^{(e x c)}\right|^{2}$, given the SP population does not participate in the process. The factor of molecular density $\rho$ signals the onset of superradiance, where the $\mathrm{PL}$ rate scales as the number of molecules in the coherently delocalized state, analogously to the situation in molecular aggregates 29. Finally, the restriction given in Eq. (2) has two consequences in Eq. (5). First, $\Theta$ turns the PL off for states to the right of the light line, and therefore for LPs and a subset of UPs, as already discussed above. Second, for a fixed value of in-plane momentum $\boldsymbol{k}$, there are at most two possible values of out-of-plane photon wavevectors $k_{z d}= \pm \sqrt{\epsilon_{d} \frac{\omega_{y_{k}}^{2}}{c^{2}}-|\boldsymbol{k}|^{2}}$, so the rate scales as the electric field $\left(\propto \omega_{y_{k}}\right)$ times the density of photonic states proportional to $\left.\frac{d k_{z d} d}{d \omega}\right|_{\omega=\omega_{y_{k}}}=\frac{\sqrt{|\boldsymbol{k}|^{2}+k_{z d}^{2}}}{c k_{d z}}=\frac{\omega_{y_{\boldsymbol{k}}}}{c k_{d z}}$; this frequency scaling is drastically different from the single-molecule case, where all photon-propagation directions are available for PL. Interestingly, there is a critical in-plane wavevector $\boldsymbol{k}^{*}=\frac{\sqrt{\epsilon_{d}} \omega_{\boldsymbol{k}^{*}}}{c}$ for which $k_{z d}=0$, which coincides with the transition between bright and non-emissive UPs. This critical propagation direction is along grazing incidence, where the emitted photon travels parallel to the metal surface. At $\boldsymbol{k}^{*},\left.\frac{d k_{z d}}{d \omega}\right|_{\omega=\omega_{y_{\boldsymbol{k}^{*}}}} \rightarrow \infty$, 

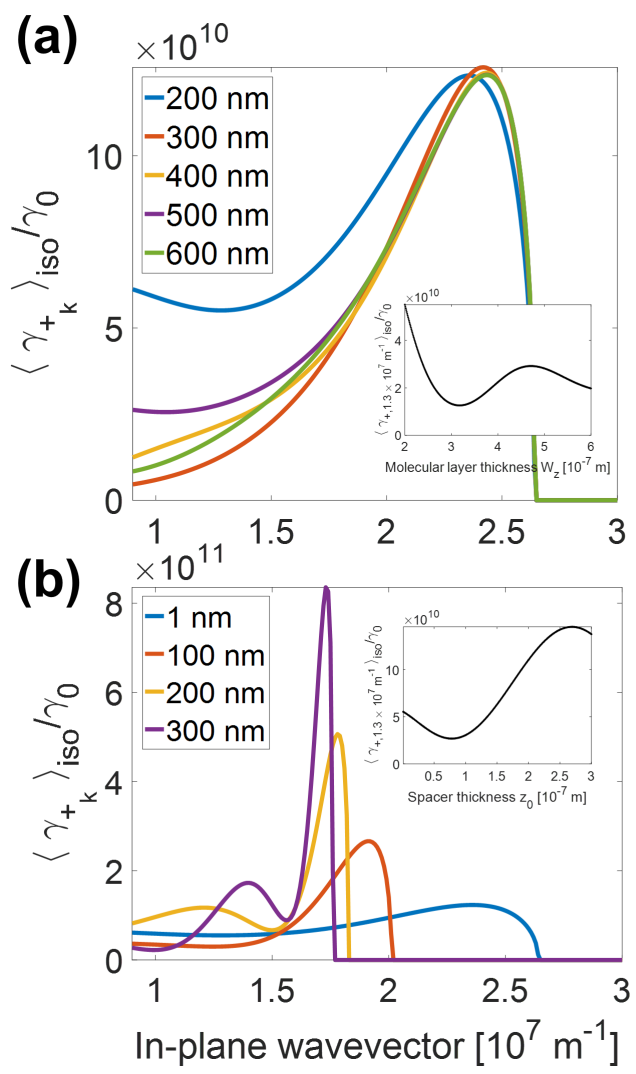

Figura 3. Normalized isotropic PL rates for the UP band $\frac{\left\langle\gamma_{+_{\boldsymbol{k}}}\right\rangle_{\text {iso }}}{\gamma_{S M}}$ as a function of (a) molecular layer and (b) spacer thickness, $W_{z}$ and $z_{0}$, respectively. Results are presented for a maximal density $\rho=3,7 \times 10^{10}$ molecules $/ \mu \mathrm{m}^{3}$. Insets trace the PL rate for $\left|+_{1,3 \times 10^{7} \mathrm{~m}^{-1}}\right\rangle$ which oscillates with $W_{z}$ and $z_{0}$. Oscillations in (a) are due to interferences of the PL processes for molecules at different heights of the layer, while oscillations in (b) are due to reflected waves from the metal.

so $\left|\left\langle\gamma_{y_{k}, 0}\right\rangle_{\text {iso }}\right|,\left|\left\langle\gamma_{y_{k}, \text { ref }}\right\rangle_{\text {iso }}\right|$ diverge, yet $r_{\boldsymbol{k}^{*}} \rightarrow-1$ (both lossless and lossy interfaces are perfectly reflective at grazing incidence, see Fig. 21). The singularities in $\left\langle\gamma_{y_{k}, i}\right\rangle_{\text {iso }}$ are van Hove anomalies [43 45] arising from the effective one-dimensional nature of the PL process where, given a fixed in-plane momentum $\boldsymbol{k}$ dictated by the SP mode, the (one-dimensional) out-of-plane momentum $k_{z d}$ must be chosen to conserve energy. Incidentally, as explained in [46], these singularities are also at the origin of the narrow resonances observed in surface lattice plasmons [47, a phenomenon described under the umbrella of RayleighWood anomalies [48, 49]. The physical origin of these collective state anomalies is thus different from single molecule emission anomalies in quasi-one-dimensional photonic crystals [50, and altogether can yield substantial enhancements of PL rates $\left(\frac{\left\langle\gamma_{y_{k}}\right\rangle_{\text {iso }}}{\gamma_{S M, 0}}>10^{9}\right)$.

The strong-coupling theory above is a good description of the plexciton system when disorder $\Sigma_{e}$ in the molecular excitation energies across the layer is small. Quantitatively, we expect our theory to fail when $\min _{y}\left|\omega_{y_{k}}-\omega_{e}\right|<$
$\Sigma_{e}$, where $\Sigma_{e} \approx 10 \mathrm{meV}$ is a typical value for organic samples [51]. That is, for the phenomenology described in this article, light-matter coupling must be strong enough that the critical point (see Fig. 2a) must lie above the bare exciton energy by at least $\Sigma_{e}$. For the parameters used in Fig. 2, our theory does not hold for plexciton and dark exciton states with $|\boldsymbol{k}|<0,9 \times 10^{7} \mathrm{~m}^{-1}$, which can efficiently form superpositions of states with various $\boldsymbol{k}$ values to localize and feature $O\left(\gamma_{S M, 0}\right)$ PL rates. On the other hand, it is known that vibrational relaxation dynamics between polaritons and dark states exhibit ultrafast relaxation rates $(\sim 50 \mathrm{fs})[20,52$. A detailed characterization of vibrational relaxation processes in molecular polaritons is a complex task on itself and beyond the scope of this work, but will be irrelevant when PL rates become much faster, such as in our case of interest. For the time being, these estimates suffice to place constraints on the applicability of the present theory. An important observation is that owing to vibrational relaxation, a much stronger $\mathrm{PL}$ is typically expected from the lower polariton branch compared to the upper one whenever the former is emissive [53. Thus, the presently described superradiant PL afforded by UPs renders plexcitonic systems unique in terms of polariton photophysics, and could be exploited to suppress photochemical pathways [54] or induce new thermodynamic equilibria [55].

Finally, we analyze other coherent effects associated with plexciton PL. Eq. (6) contains finite Fourier transforms of the coupling $J_{\boldsymbol{k}}(z)$ at the wavevector $k_{z d}$, which arise from interferences of emission pathways due to molecules at different heights of the layer. This implies oscillatory features of $\left\langle\gamma_{y_{k}, i}\right\rangle_{\text {iso }}$ as a function of molecular layer thickness $W_{z}$ across an $O\left(k_{z d}^{-1}\right)$ range of values. As explained in the previous paragraph, our theory is valid for strong coupling, and therefore, close to the anticrossing region and the critical wavevector $\boldsymbol{k}=\boldsymbol{k}^{*}$, so $k_{d z}$ is typically small and this oscillatory effect is not appreciable unless $W_{z}$ is very big $(>1 \mu \mathrm{m})$ or if $\rho$ increases. To see this oscillatory effect, we increase the density to a maximal value $\rho=3,7 \times 10^{10}$ molecules $/ \mu \mathrm{m}^{3}$, associated with the minimum van der Waals contact distance between chromophores, and obtain Fig. 3 a. The inset shows results for a superradiant state $\left|+1,3 \times 10^{7} \mathrm{~m}^{-1}\right\rangle$, which exhibits PL oscillations as a function of $W_{z}$ within the first $600 \mathrm{~nm}$. It is also interesting to examine yet another oscillatory behavior of $\left\langle\gamma_{y_{k}, i}\right\rangle_{\text {iso }}$ obtained by varying the spacer thickness $z_{0}$. This effect is due exclusively to interference between incident and reflected waves, and therefore, occurs when $z_{0}$ varies across photonic distances, by analogy to the aforementioned Drexhage [1, 2] and CPS [3, 4, problem. Since we want to stay in the strong coupling regime, we keep the maximal $\rho$ while varying $z_{0}$ up to a threshold $\sim 400 \mathrm{~nm}$, after which light-matter coupling becomes weak for the wavevectors of interest. Fig. 3b shows the oscillatory behavior of the PL for $\left|+{ }_{1,3 \times 10^{7} \mathrm{~m}^{-1}}\right\rangle$ as a function of $z_{0}$.

To conclude, we have presented a comprehensive quantum formalism to study the PL properties of plexciton 
systems, thus generalizing the paradigmatic problem of single-molecule PL next to a metal interface. We have shown there is rich phenomenology associated with superradiance and the transition between bright and nonemissive plexciton states. Finally, we have elucidated two types of coherent effects exhibited by plexciton PL at high molecular densities. These include generalizations of the well-known PL oscillations due to reflected waves from the metal, but also new coherences that arise from multiple emission pathways corresponding to different distances of the molecules from the metal layer (a result of finite thickness of the molecular layer). The described properties can be readily verified in experiments and present new control knobs to manipulate and design photophysical properties in molecular materials.

J.Y.Z. acknowledges discussions with Misha Fogler, Jean-Jacques Greffet, Nicholas Rivera, Matthew Du, and Raphael Ribeiro, as well as funding from NSF CAREER CHE 1654732 as well as UCSD startup. S.K.S. acknowledges support from the Center for Excitonics, an Energy Frontier Research Center funded by the U.S. Department of Energy under Award de-sc0001088 and the Ministry of Education and Science of the Russian Federation for supporting the research in the framework of the state assignment, award \#3.2166.2017/4.6. V.M. acknowledges funding from U. S. Department of Energy under Award de-sc0017760
[1] K. H. Drexhage, H. Kuhn, and F. P. Schäfer. Variation of the fluorescence decay time of a molecule in front of a mirror. Ber. Bunsenges. Phys. Chem., 72(2):329-329, 1968.

[2] K. H. Drexhage. Influence of a dielectric interface on fluorescence decay time. J. Lumin., 1:693-701, 1970.

[3] R. R. Chance, A. Prock, and R. Silbey. Lifetime of an emitting molecule near a partially reflecting surface. $J$. Chem. Phys., 60(7):2744-2748, 1974.

[4] R. R. Chance, A. Prock, and R. Silbey. Molecular fluorescence and energy transfer near interfaces. Adv. Chem. Phys., 37(1):65, 1978.

[5] A. Sommerfeld. Über die ausbreitung der wellen in der drahtlosen telegraphie. Ann. Phys., 333(4):665-736, 1909.

[6] A. Gonz $\widetilde{A}_{j}$ lez-Tudela, P. A. Huidobro, L. Mart $\tilde{A} n-$ Moreno, C. Tejedor, and F. J. Garc Âa-Vidal. Theory of strong coupling between quantum emitters and propagating surface plasmons. Phys. Rev. Lett., 110:126801, Mar 2013.

[7] P. Törmä and W.L. Barnes. Strong coupling between surface plasmon polaritons and emitters: a review. Rep. Prog. Phys., 78(1):013901, 2015.

[8] J. Bellessa, C. Bonnand, J. C. Plenet, and J. Mugnier. Strong coupling between surface plasmons and excitons in an organic semiconductor. Phys. Rev. Lett., 93:036404, Jul 2004.

[9] N. T. Fofang, N. K. Grady, Z. Fan, A. O. Govorov, and N. J. Halas. Plexciton dynamics: Exciton-plasmon coupling in a j-aggregate-au nanoshell complex provides a mechanism for nonlinearity. Nano Lett., 11(4):15561560, 2011.

[10] T. Ozel, P. L. Hernandez-Martinez, E. Mutlugun, O. Akin, S. Nizamoglu, I. O. Ozel, Q. Zhang, Q. Xiong, and H. V. Demir. Observation of selective plasmonexciton coupling in nonradiative energy transfer: donorselective versus acceptor-selective plexcitons. Nano Lett., 13(7):3065-3072, 2013.

[11] S. T. Kochuveedu and D. H. Kim. Surface plasmon resonance mediated photoluminescence properties of nanostructured multicomponent fluorophore systems. Nanoscale, 6(10):4966-4984, 2014.

[12] J. Yuen-Zhou, S. S. Saikin, T. Zhu, M. C. Onbasli, C. A. Ross, V. Bulovic, and M. A. Baldo. Plexciton dirac points and topological modes. Nat. Commun., 7(11783), 2016.

[13] P. W. Brumer and M. Shapiro. Principles of the Quantum Control of Molecular Processes. Wiley-Interscience, 2003.

[14] T. W. Ebbesen. Hybrid light-matter states in a molecular and material science perspective. Acc. Chem. Res., 49 (11):2403-2412, 2016.

[15] F. Herrera and F. C. Spano. Cavity-controlled chemistry in molecular ensembles. Phys. Rev. Lett., 116(23):238301, 2016.

[16] J. Galego, F. J. Garcia-Vidal, and J. Feist. Suppressing photochemical reactions with quantized light fields. Nat. Comms., 7, 2016.

[17] J. Flick, M. Ruggenthaler, H. Appel, and A. Rubio. Atoms and molecules in cavities, from weak to strong coupling in quantum-electrodynamics (qed) chemistry. Proc. Nac. Acad. Sci., 114(12):3026-3034, 2017.

[18] D. G. Baranov, M. Wersall, J. Cuadra, T. J. Antosiewicz, and T. Shegai. Novel nanostructures and materials for strong light-matter interactions. ACS Photon., 5(1):24$42,2017$.

[19] M. Sukharev and A. Nitzan. Optics of exciton-plasmon nanomaterials. J. Phys.: Condens. Matter, 29(44): 443003, 2017.

[20] R. F. Ribeiro, L. A. Martínez-Martínez, M. Du, J. Campos-González-Angulo, and J. Yuen-Zhou. Polariton chemistry: controlling molecular dynamics with optical cavities. Chem. Sci., 9:6325-6339, 2018.

[21] X. Zhong, T. Chervy, L. Zhang, A. Thomas, J. George, C. Genet, J. A. Hutchison, and T. W. Ebbesen. Energy transfer between spatially separated entangled molecules. Angew. Chem., Int. Ed., 56(31):9034-9038, 2017.

[22] M. Du, L. A. Martínez-Martínez, R. F. Ribeiro, Z. Hu, V. Menon, and J. Yuen-Zhou. Theory for polaritonassisted remote energy transfer. Chem. Sci., 9:6359-6669, 2018.

[23] R. Sáez-Blázquez, J. Feist, A. I. Fernández-Domínguez, and F. J. García-Vidal. Organic polaritons enable local vibrations to drive long-range energy transfer. Phys. Rev. B, 97(24):241407, 2018.

[24] W. Gao, X. Li, M. Bamba, and J. Kono. Continuous transition between weak and ultrastrong coupling through exceptional points in carbon nanotube microcavity exciton-polaritons. Nat. Photon., 12:362-367, 2018.

[25] E. R. Bittner and C. Silva. Estimating the conditions for 
polariton condensation in organic thin-film microcavities. J. Chem. Phys., 136(3):034510, 2012.

[26] K.S. Daskalakis, S.A. Maier, R. Murray, and S. KénaCohen. Nonlinear interactions in an organic polariton condensate. Nat. Mater., 13(3):271-278, 2014.

[27] S. Zaster, E. R. Bittner, and A. Piryatinski. Quantum symmetry breaking of exciton/polaritons in a metalnanorod plasmonic array. J. Phys. Chem. A, 120(19): 3109-3116, 2016.

[28] R. H. Dicke. Coherence in spontaneous radiation processes. Phys. Rev., 93(1):99, 1954.

[29] F. C. Spano and S. Mukamel. Superradiance in molecular aggregates. J. Chem. Phys., 91(2):683-700, 1989.

[30] S. A. Maier. Plasmonics: Fundamentals and Applications. Springer, 2007.

[31] L. Novotny and B. Hecht. Principles of Nano-Optics. Cambridge University Press, 2012.

[32] P. K. Aravind and H. Metiu. Use of a perfectly conducting sphere to excite the plasmon of a flat surface. 1. calculation of the local field with applications to surfaceenhanced spectroscopy. J. Phys. Chem., 86(26):50765084, 1982.

[33] G. C. Schatz and M. A. Ratner. Quantum mechanics in chemistry. Dover, 2002.

[34] M. O Scully and M. S. Zubairy. Quantum optics. Cambridge University Press, 1997.

[35] H. F. Arnoldus and T. F. George. Spontaneous decay and atomic fluorescence near a metal surface or an absorbing dielectric. Phys. Rev. A, 37(3):761, 1988.

[36] G. W. Ford and W. H. Weber. Electromagnetic interactions of molecules with metal surfaces. Phys. Rep., 113 (4):195-287, 1984.

[37] WL Barnes. Fluorescence near interfaces: the role of photonic mode density. J. Mod. Opt., 45(4):661-699, 1998.

[38] J. A. Kong. Theory of electromagnetic waves. WileyInterscience, 1975.

[39] C.-T. Tai. Dyadic Green functions in electromagnetic theory. Institute of Electrical \& Electronics Engineers (IEEE), 1994.

[40] H. T. Dung, L. Knöll, and D.-G. Welsch. Spontaneous decay in the presence of dispersing and absorbing bodies: General theory and application to a spherical cavity. Phys. Rev. A, 62(5):053804, 2000.

[41] W. Vogel and D.-G. Welsch. Quantum optics. John Wiley \& Sons, 2006.

[42] S. Scheel and S. Buhmann. Macroscopic quantum electrodynamics-concepts and applications. Acta Phys. Slovaca, 58(5):675-809, 2008.

[43] L. Van Hove. The occurrence of singularities in the elastic frequency distribution of a crystal. Phys. Rev., 89(6): 1189, 1953.

[44] L. C. Andreani, F. Tassone, and F. Bassani. Radiative lifetime of free excitons in quantum wells. Solid State Commun., 77(9):641-645, 1991.

[45] M. Remeika, J. R. Leonard, C. J. Dorow, M. M. Fogler, L. V. Butov, M. Hanson, and A. C. Gossard. Measurement of exciton correlations using electrostatic lattices. Phys. Rev. B, 92:115311, 2015.

[46] F. J. García de Abajo. Colloquium: Light scattering by particle and hole arrays. Rev. Mod. Phys., 79(4):1267, 2007.

[47] S. Zou, N. Janel, and G. C. Schatz. Silver nanoparticle array structures that produce remarkably narrow plasmon lineshapes. J. Chem. Phys., 120(23):10871-10875,
2004.

[48] R. W. Wood. Anomalous diffraction gratings. Phys. Rev., 48(12):928, 1935.

[49] U. Fano. The theory of anomalous diffraction gratings and of quasi-stationary waves on metallic surfaces. $J$. Opt. Soc. Amer., 31(3):213-222, 1941.

[50] E. Viasnoff-Schwoob, C. Weisbuch, H. Benisty, S. Olivier, S. Varoutsis, I. Robert-Philip, R. Houdré, and C. J. M. Smith. Spontaneous emission enhancement of quantum dots in a photonic crystal wire. Phys. Rev. Lett., 95(18): 183901, 2005.

[51] S. Jang, S. E. Dempster, and R. J. Silbey. Characterization of the static disorder in the b850 band of lh2. J. Phys. Chem. B, 105(28):6655-6665, 2001.

[52] V. M. Agranovich, M. Litinskaia, and D. G. Lidzey. Cavity polaritons in microcavities containing disordered organic semiconductors. Phys. Rev. B, 67(8):085311, February 2003.

[53] D. G. Lidzey, D. D. C. Bradley, T. Virgili, A. Armitage, M. S. Skolnick, and S. Walker. Room temperature polariton emission from strongly coupled organic semiconductor microcavities. Phys. Rev. Lett., 82:3316-3319, Apr 1999.

[54] J. A. Hutchison, T. Schwartz, C. Genet, E. Devaux, and T. W. Ebbesen. Modifying chemical landscapes by coupling to vacuum fields. Angew. Chem. Int. Ed. Engl., 51(7):1592-1596, 2012.

[55] A. Thomas, J. George, A. Shalabney, M. Dryzhakov, S. J. Varma, J. Moran, T. Chervy, X. Zhong, E. Devaux, C. Genet, J. A. Hutchison, and T. W. Ebbesen. Groundstate chemical reactivity under vibrational coupling to the vacuum electromagnetic field. Angew. Chem. Int., 128(38):11634-11638, 2016.

[56] A. Archambault, F. Marquier, J.-J. Greffet, and C. Arnold. Quantum theory of spontaneous and stimulated emission of surface plasmons. Phys. Rev. B, 82:035411, Jul 2010.

[57] Note1. The final result in Eq. S8 is twice what is reported in the Supplementary Material of [56], although we believe our result here [12] is correct.

[58] Note2. (Notice that the expression for the isotropic collective coupling reported in 6] (two paragraphs after Eq. 4 , it reads $\left.g_{\text {iso }}^{2}=\frac{2}{3} g_{\|}^{2}+\frac{1}{3} g_{\perp}^{2}\right)$ is incorrect; it should read $\left.g_{\text {iso }}^{2}=\frac{1}{3} g_{\|}^{2}+\frac{1}{3} g_{\perp}^{2}\right)$.

[59] W. Heitler. The quantum theory of radiation. Courier Corporation, 1954.

[60] J. M. Wylie and J. E. Sipe. Quantum electrodynamics near an interface. Phys. Rev. A, 30:1185-1193, Sep 1984.

[61] P.R. West, S. Ishii, G.V. Naik, N.K. Emani, V.M. Shalaev, and A. Boltasseva. Searching for better plasmonic materials. Laser Photon. Rev., 4(6):795-808, 2010. 


\title{
Supplemental Material for "Molecular emission near metal interfaces: the polaritonic regime"
}

\author{
Joel Yuen-Zhou ${ }^{1}$, Semion K. Saikinn ${ }^{2,3}$, Vinod M. Menon ${ }^{4}$ \\ ${ }^{1}$ Department of Chemistry and Biochemistry, University of California San Diego, La Jolla, CA, USA. \\ ${ }^{2}$ Department of Chemistry and Chemical Biology, Harvard University, Cambridge, MA, USA. \\ ${ }^{3}$ Institute of Physics, Kazan Federal University, Kazan, Russian Federation. \\ ${ }^{4}$ Department of Physics, Graduate Center and City College of New York, City University of New York, New York, \\ New York, USA.
}

joelyuen@ucsd.edu

Section I considers the quantization of lossless SP modes, which is utilized in the plexciton Hamiltonian of Eq. (1) (derived in Section II). Since PL rates are derived within a Fermi golden rule approach, neglect of losses amounts to regarding the initial and final states in Eq. (3) to be eigenstates of Eq. (1), which offers a good zeroth-order description of the plexciton setup in the strong coupling regime, where the linewidth due to metal loss is much smaller than the Rabi splitting. Derivations of PL rates within the modal representation and the dyadic Green's function formalism are carried out in Section II Finally, in Section IV] the PL expressions are shown, within the formalism of M-QED, to agree with the case where metal losses are taken into account in the UHP photonic density of states.

\section{SP MODES: PROPERTIES AND QUANTIZATION}

In this Section, we present the main steps to quantize lossless SP modes. For further details, the reader might wish to consult [56] or our previous work [12], in particular, its Supplementary Note 1.

\section{A. Properties of SP modes}

We summarize the main features of SP modes arising at the interface between a dielectric material $d(z>0)$ and a (Drude) metal $m(z<0)$, each with relative permittivities $\epsilon_{d}$ and $\epsilon_{m}(\omega)=\epsilon_{\infty}-\frac{\omega_{P}^{2}}{\omega^{2}+i \omega \Gamma}$, where $\omega_{P}$ is the plasma frequency and $\Gamma$ is a damping constant [30, 31] (we hereafter set $\Gamma=0$, and revisit the effects of metal losses in Section IV]. The modes are labeled by an in-plane wavevector $\boldsymbol{k}$ and are characterized by the following electromagnetic fields,

$$
\begin{aligned}
\vec{E}(\boldsymbol{k}) & =\mathcal{A}_{\boldsymbol{k}} \boldsymbol{E}(\boldsymbol{k}) e^{i\left(\boldsymbol{k} \cdot \boldsymbol{r}-\omega_{\boldsymbol{k}}^{S P} t\right)}, \\
\vec{B}(\boldsymbol{k}) & =\mathcal{A}_{\boldsymbol{k}} \boldsymbol{B}(\boldsymbol{k}) e^{i\left(\boldsymbol{k} \cdot \boldsymbol{r}-\omega_{k}^{S P} t\right)} .
\end{aligned}
$$

where $\mathcal{A}_{\boldsymbol{k}}$ is a mode amplitude (see Section $\mathrm{IB}$. The frequencies corresponding to each of these modes are $\omega_{\boldsymbol{k}}^{S P}=c \Omega_{\boldsymbol{k}}^{S P}$, where,

$$
\begin{aligned}
\Omega_{\boldsymbol{k}}^{S P} & =k \sqrt{\frac{\epsilon_{d}+\epsilon_{m}}{\epsilon_{d} \epsilon_{m}}} \\
& =\sqrt{\frac{\epsilon_{d} \Omega_{P}^{2}+k^{2}\left(\epsilon_{d}+\epsilon_{\infty}\right)-\sqrt{\left[\epsilon_{d} \Omega_{P}^{2}+k^{2}\left(\epsilon_{d}+\epsilon_{\infty}\right)\right]^{2}-4 \epsilon_{\infty} \epsilon_{d} k^{2} \Omega_{P}^{2}}}{2 \epsilon_{\infty} \epsilon_{d}}}
\end{aligned}
$$

This equation was used to plot the SP dispersion band in Fig. 2a. Another way to write it is, 


$$
\Omega_{\boldsymbol{k}}^{S P}=\frac{\sqrt{|\boldsymbol{k}|^{2}+k_{z d}^{2}}}{\sqrt{\epsilon_{d}}}=\frac{\sqrt{|\boldsymbol{k}|^{2}+k_{z m}^{2}}}{\sqrt{\epsilon_{m}}} .
$$

Eq. S3 implies that for SP modes, the vertical components of the wavevector in the dielectric $\left(k_{z d}\right)$ and the metal $\left(k_{z m}\right)$ depend on $|\boldsymbol{k}|=k$. Since they represent evanescent decays, we write $k_{z d}=i \alpha_{d k}$ and $k_{z m}=-i \alpha_{m k}$, where $\alpha_{d k}, \alpha_{m k} \in \Re$ and positive. The vector components of the modes in Eq. $\mathrm{S} 1$ are given by,

$$
\begin{aligned}
& \boldsymbol{E}=\mathcal{A}_{\boldsymbol{k}}\left[\Theta(-z) \boldsymbol{E}_{m} e^{\alpha_{m k} z}+\Theta(z) \boldsymbol{E}_{d} e^{-\alpha_{d k} z}\right], \\
& \boldsymbol{B}=\mathcal{A}_{\boldsymbol{k}}\left[\Theta(-z) \boldsymbol{B}_{m} e^{\alpha_{m k} z}+\Theta(z) \boldsymbol{B}_{d} e^{-\alpha_{d k} z}\right],
\end{aligned}
$$

and each of the involved vectors are given by

$$
\begin{aligned}
& \boldsymbol{E}_{d}=\left(1,0, \frac{i k}{\alpha_{d k}}\right), \\
& \boldsymbol{B}_{d}=\left(0,-\frac{i \omega_{\boldsymbol{k}}^{S P} \epsilon_{d}}{\alpha_{d k} c^{2}}, 0\right)
\end{aligned}
$$

and

$$
\begin{aligned}
& \boldsymbol{E}_{m}=\left(1,0,-\frac{i k}{\alpha_{m k}}\right), \\
& \boldsymbol{B}_{m}=\left(0, \frac{i \omega_{\boldsymbol{k}}^{S P} \epsilon_{m}}{\alpha_{m k} c^{2}}, 0\right) .
\end{aligned}
$$

where the Cartesian notation we are using in this Section is expressed using the unit vectors $\hat{\boldsymbol{k}}, \hat{\boldsymbol{\theta}}_{\boldsymbol{k}}, \hat{\boldsymbol{z}}$ such that $\hat{\boldsymbol{k}} \times \hat{\boldsymbol{\theta}}_{\boldsymbol{k}}=\hat{\boldsymbol{z}}$, so that $\boldsymbol{E}=\left(E_{k}, E_{\theta_{k}}, E_{z}\right)$, where $E_{i}=\boldsymbol{E} \cdot \hat{\boldsymbol{i}}$ (note that the tangential direction of $\hat{\boldsymbol{\theta}}_{\boldsymbol{k}}$ is with respect to $\hat{\boldsymbol{k}}$ and not to $\hat{\boldsymbol{r}}$ ). Beware that we are using a different Cartesian notation than the one in the main text.

\section{B. Quantization of SP modes}

The energy in the $\boldsymbol{k}$ th mode in Eq. [S1] is quadratic in the fields [31, 56,

$$
H_{S P, \boldsymbol{k}}=\frac{1}{2} \sum_{i} \int d V\left[\epsilon_{0} \sum_{j} \frac{d\left(\omega \epsilon^{*}(\omega)\right)}{d \omega}\left|(\vec{E})_{j}\right|^{2}+\frac{1}{\mu_{0} \mu}\left|(\vec{B})_{i}\right|^{2}\right]\left|\mathcal{A}_{\boldsymbol{k}}\right|^{2},
$$

where $i, j \in\left\{k, \theta_{k}, z\right\}$. Taking the integration volume to be a box of in-plane area $S$ and infinite height, we plug in Eq. S5 into Eq. S6 and use $\int d x d y=S, \int_{-\infty}^{0} d z e^{-2 \alpha_{d} z}=\frac{1}{2 \alpha_{d}}$, and $\int_{-\infty}^{0} d z e^{-2 \alpha_{m} z}=\frac{1}{2 \alpha_{m}}$ to obtain,

$$
\begin{aligned}
H_{S P, \boldsymbol{k}} & =S\left|\mathcal{A}_{\boldsymbol{k}}\right|^{2} \sum_{i}\left\{\left[\epsilon_{0} \epsilon_{d}\left|E_{i, d}\right|^{2}+\frac{1}{\mu_{0} \mu}\left|B_{i, d}\right|^{2}\right] \frac{1}{4 \alpha_{d k}}+\left[\epsilon_{0} \frac{d\left(\omega \epsilon_{m}(\omega)\right)}{d \omega}\left|E_{i, m}\right|^{2}+\frac{1}{\mu_{0} \mu}\left|B_{i, m}\right|^{2}\right] \frac{1}{4 \alpha_{m k}}\right\} \\
& =S \frac{\epsilon_{0} L_{\boldsymbol{k}}}{4}\left(\mathcal{A}_{\boldsymbol{k}} \mathcal{A}_{\boldsymbol{k}}^{*}+\mathcal{A}_{\boldsymbol{k}}^{*} \mathcal{A}_{\boldsymbol{k}}\right),
\end{aligned}
$$

where the mode length $L_{\boldsymbol{k}}[12,56]$ is defined as,

$$
\begin{aligned}
L_{\boldsymbol{k}} & =\sum_{i}\left\{\left[\epsilon_{0} \epsilon_{d}\left|E_{i, d}\right|^{2}+\frac{1}{\mu_{0} \mu}\left|B_{i, d}\right|^{2}\right] \frac{1}{2 \epsilon_{0} \alpha_{d k}}+\left[\left.\epsilon_{0} \frac{d\left(\omega \epsilon_{m}(\omega)\right)}{d \omega}\right|_{\omega=\frac{\Omega_{0}}{c}}\left|E_{i, m}\right|^{2}+\frac{1}{\mu_{0} \mu}\left|B_{i, m}\right|^{2}\right] \frac{1}{2 \epsilon_{0} \alpha_{m k}}\right\} \\
& =\frac{-\epsilon_{m}}{\alpha_{d k}}+\frac{1}{2 \alpha_{m k}}\left[\left.\frac{d\left(\omega \epsilon_{m}(\omega)\right)}{d \omega}\right|_{\omega=\frac{\Omega(\boldsymbol{k})}{c}}\left(\frac{\epsilon_{m}-\epsilon_{d}}{\epsilon_{m}}\right)-\epsilon_{m}-\epsilon_{d}\right] .
\end{aligned}
$$


In this derivation, we have used $\mu=1, \epsilon_{0} \mu_{0}=c^{-2}$ as well as Eqs. (S2) and [S3) [57].

Eq. (S7) is quadratic in the electric field amplitude $\mathcal{A}_{\boldsymbol{k}}$, so each $\boldsymbol{k}$ mode corresponds to a harmonic oscillator,

$$
H_{S P, \boldsymbol{k}}=\frac{\hbar \omega_{\boldsymbol{k}}^{S P}}{2}\left(\zeta_{\boldsymbol{k}} \zeta_{\boldsymbol{k}}^{*}+\zeta_{\boldsymbol{k}}^{*} \zeta_{\boldsymbol{k}}\right)
$$

so that the amplitude $\mathcal{A}_{\boldsymbol{k}}$ can be related to $\zeta_{\boldsymbol{k}}$ by,

$$
\mathcal{A}_{k}=\sqrt{\frac{2 \hbar \omega_{k}^{S P}}{\epsilon_{0} S L_{k}}} \zeta_{k}
$$

Promoting the complex amplitudes to SP annhilation and creation operators, $\zeta_{\boldsymbol{k}} \rightarrow a_{\boldsymbol{k}}$ and $\zeta_{\boldsymbol{k}}^{*} \rightarrow a_{\boldsymbol{k}}^{\dagger}$ with $\left[a_{\boldsymbol{k}}, a_{\boldsymbol{k}}^{\dagger}\right]=1$,

$$
\begin{aligned}
H_{S P, \boldsymbol{k}} & =\frac{\hbar \omega_{\boldsymbol{k}}^{S P}}{2}\left(a_{\boldsymbol{k}} a_{\boldsymbol{k}}^{\dagger}+a_{\boldsymbol{k}}^{\dagger} a_{\boldsymbol{k}}\right) \\
& =\frac{\hbar \omega_{\boldsymbol{k}}^{S P}}{2}\left(a_{\boldsymbol{k}}^{\dagger} a_{\boldsymbol{k}}+\frac{1}{2}\right) .
\end{aligned}
$$

The Hamiltonian $H_{S P}=\sum_{\boldsymbol{k}} H_{S P, \boldsymbol{k}}-\sum_{\boldsymbol{k}} \frac{\hbar \omega_{\boldsymbol{k}}^{S P}}{2}=\hbar \omega_{\boldsymbol{k}}^{S P} a_{\boldsymbol{k}}^{\dagger} a_{\boldsymbol{k}}$ in the main text ignores the background zero-point energies of the SP modes since the energy $\omega_{e}$ of the excitons already takes this background into account.

The electric field and magnetic induction are superpositions of amplitudes in each of the $\boldsymbol{k}$ modes,

$$
\begin{aligned}
\overrightarrow{\mathcal{E}} & =\sum_{\boldsymbol{k}} \mathcal{A}_{\boldsymbol{k}} \vec{E}(\boldsymbol{k}) . \\
\overrightarrow{\mathcal{B}} & =\sum_{\boldsymbol{k}} \mathcal{A}_{\boldsymbol{k}} \vec{B}(\boldsymbol{k}) .
\end{aligned}
$$

Promoting these amplitudes to quantum operators in the Heisenberg picture, and using Eq. S10],

$$
\begin{aligned}
\hat{\mathcal{E}}(\boldsymbol{r}, t) & =\sum_{\boldsymbol{k}} \sqrt{\frac{\hbar \omega_{\boldsymbol{k}}^{S P}}{2 \epsilon_{0} S L_{\boldsymbol{k}}}} a_{\boldsymbol{k}} \vec{E}(\boldsymbol{k})+\sqrt{\frac{\hbar \omega_{\boldsymbol{k}}^{S P}}{2 \epsilon_{0} S L_{\boldsymbol{k}}}} a_{\boldsymbol{k}}^{\dagger} \vec{E}^{*}(\boldsymbol{k}), \\
\hat{\mathcal{B}}(\boldsymbol{r}, t) & =\sum_{\boldsymbol{k}} \sqrt{\frac{\hbar \omega_{\boldsymbol{k}}^{S P}}{2 \epsilon_{0} S L_{\boldsymbol{k}}}} a_{\boldsymbol{k}} \vec{B}(\boldsymbol{k})+\sqrt{\frac{\hbar \omega_{\boldsymbol{k}}^{S P}}{2 \epsilon_{0} S L_{\boldsymbol{k}}}} a_{\boldsymbol{k}}^{\dagger} \vec{B}^{*}(\boldsymbol{k}),
\end{aligned}
$$

where we have used the fact that $\mathcal{E}$ and $\boldsymbol{B}$ are real valued to write the results in a symmetrized fashion.

In the main text, the electric field in the Schrodinger picture for the dielectric region $z>0$ is,

$$
\hat{\mathcal{E}}\left(\boldsymbol{r}_{\boldsymbol{n}}\right)=\sum_{\boldsymbol{k}} \sqrt{\frac{\hbar \omega_{\boldsymbol{k}}^{S P}}{2 \epsilon_{0} S L_{\boldsymbol{k}}}} a_{\boldsymbol{k}} \underbrace{\left(1,0, \frac{i k}{\alpha_{d k}}\right)}_{=\boldsymbol{E}_{\boldsymbol{k}}=\hat{\boldsymbol{k}}+\frac{i|\boldsymbol{k}|}{\alpha_{d k}} \hat{\boldsymbol{z}}} e^{i \boldsymbol{k} \cdot \boldsymbol{r}_{n}-\alpha_{d} z_{s}}+\text { h.c. }
$$

\section{DERIVATION OF PLEXCITON HAMILTONIAN, EQ. (1)}

In this Section, we abound on the quantum-mechanical formalism to describe the plexciton system and ultimately leading to Eq. (1) in the main text. The Hamiltonian $H$ for this setup of interest can be expressed as

$$
H=H_{e x c}+H_{S P}+H_{e x c-S P},
$$


where $H_{e x c}=\sum_{\boldsymbol{n}, s} \hbar \omega_{\boldsymbol{n} s}^{(e)} \sigma_{\boldsymbol{n} s}^{\dagger} \sigma_{\boldsymbol{n} s}, H_{S P}=\sum_{\boldsymbol{k}} H_{\boldsymbol{k}}=\sum_{\boldsymbol{k}} \hbar \omega_{\boldsymbol{k}}^{S P} a_{\boldsymbol{k}}^{\dagger} a_{\boldsymbol{k}}$, and $H_{e x c-S P}=-\sum_{\boldsymbol{n}, s} \hat{\boldsymbol{\mu}}_{\boldsymbol{n} s} \cdot \hat{\mathcal{E}}\left(\boldsymbol{r}_{\boldsymbol{n} s}\right)$ denote the energetics of the molecular exciton layer, the SP modes of the metal, and the dipolar coupling between them; we neglect dipole-dipole hopping interactions between exciton states, since they play a minor role in our problem compared to light-matter coupling. We consider a layer containing $N=N_{x} N_{y} N_{z}$ emitters, with $N_{i}$ molecules along each axis $i$; $\boldsymbol{n}=\left(n_{x}, n_{y}\right)$ is a Cartesian pair of integers denoting the in-plane location $\boldsymbol{r}_{\boldsymbol{n}}=\boldsymbol{n} \cdot\left(\Delta_{x}, \Delta_{y}\right)$ of a chromophore, while the integer $s$ denotes its vertical location $z_{s} \hat{z}$ at $z_{s}=z_{0}+s \Delta_{z} ; z=z_{0}>0$ and $z=z_{f}$ label the lowest and highest vertical coordinate for a molecule in the layer and $z=0$ refers to the metal-dielectric spacer interface. $W_{z}=N_{z} \Delta_{z}$ is the vertical thickness of the molecular layer. With this convention, the location of the chromophore labeled $\boldsymbol{n} s$ is $\boldsymbol{r}_{\boldsymbol{n} s}=\boldsymbol{r}_{n}+\left(z_{0}+s \Delta_{z}\right) \hat{\boldsymbol{z}}$; its corresponding single(-Frenkel)-exciton energy and creation (annhilation) operator are $\hbar \omega_{\boldsymbol{n} s}$ and $\sigma_{\boldsymbol{n} s}^{\dagger}\left(\sigma_{\boldsymbol{n} s}\right)$. Thus, the dipole operator reads as $\hat{\boldsymbol{\mu}}_{\boldsymbol{n} s}=\boldsymbol{\mu}_{\boldsymbol{n} s}\left(\sigma_{\boldsymbol{n} s}^{\dagger}+\sigma_{\boldsymbol{n} s}\right)$. The parameters for $H_{S P}$ and $\hat{\mathcal{E}}\left(\boldsymbol{r}_{\boldsymbol{n} s}\right)$ have been defined in the previous Section.

For simplicity, we first consider identical exciton transitions $\left(\omega_{\boldsymbol{n} s}=\omega_{e}, \boldsymbol{\mu}_{\boldsymbol{n} s}=\boldsymbol{\mu}_{e g}\right)$. This is a good approximation because we are mainly interested in the "anticrossing" region, where light and matter couple much more strongly than the energetic disorder due to imperfections of the molecular layer; the extension to an isotropically oriented sample is straightforward [6]. We also simplify the problem by invoking the rotating-wave approximation (ignore "off-resonant" $\sigma_{\boldsymbol{n} s}^{\dagger} a_{\boldsymbol{k}}^{\dagger}$ and $\sigma_{\boldsymbol{n} s} a_{\boldsymbol{k}}$ terms in $H_{\text {exc-SP }}$ [34. Given these assumptions, translational invariance allows for a Bloch decomposition of the Hamiltonian in Eq. (S15) as $H=\sum_{\boldsymbol{k}} H_{\boldsymbol{k}}$, where $H_{\boldsymbol{k}}$ is given by Eq. (1). In that expression, we have defined $N_{x} N_{y}$ collective $\boldsymbol{k}=\left(k_{x}, k_{y}\right)$ exciton states $\left(k_{i}=\frac{2 \pi m_{i}}{N_{i} \Delta_{i}}\right.$ for $\left.m_{i}=-\frac{N_{i}}{2}, \cdots, \frac{N_{i}}{2}-1\right)$ represented by the operators $\sigma_{\boldsymbol{k}}^{\dagger}=\sqrt{\frac{N_{x} N_{y}}{S}} \frac{\sum_{s} J_{\boldsymbol{k}}\left(z_{s}\right)}{\mathcal{J}_{\boldsymbol{k}}} \sigma_{\boldsymbol{k} s}^{\dagger}[6]$, where $\sigma_{\boldsymbol{k} s}^{\dagger}=\frac{\sum_{\boldsymbol{n}} \sigma_{\boldsymbol{n} s}^{\dagger} e^{i \boldsymbol{k} \cdot \boldsymbol{r}_{\boldsymbol{n}}}}{\sqrt{N_{x} N_{y}}}, J_{\boldsymbol{k}}(z)=\sqrt{S}\left(\sqrt{\frac{\hbar \omega_{\boldsymbol{k}}^{S P}}{2 \epsilon_{0} S L_{\boldsymbol{k}}}} \boldsymbol{\mu}_{e g} \cdot \boldsymbol{E}_{\boldsymbol{k}} e^{-\alpha_{\boldsymbol{k}} z}\right)$, and $\mathcal{J}_{\boldsymbol{k}}=\sqrt{\rho \int_{z_{0}}^{z_{f}} d z\left|J_{\boldsymbol{k}}(z)\right|^{2}}$ is an effective collective coupling, with $\rho$ being the density of molecules in the layer. It follows that we can write $\sigma_{\boldsymbol{k}}^{\dagger}=\sum_{\boldsymbol{n}, s} d_{\boldsymbol{n} s} \sigma_{\boldsymbol{n} s}^{\dagger}$, where

$$
d_{\boldsymbol{n} s}=\sqrt{\frac{1}{S}} \frac{\sum_{s} J_{\boldsymbol{k}}\left(z_{s}\right)}{\mathcal{J}_{\boldsymbol{k}}} e^{i \boldsymbol{k} \cdot \boldsymbol{r}_{\boldsymbol{n}}} .
$$

$H_{\text {dark }, \boldsymbol{k}}=\hbar \omega_{e}\left(\mathbb{I}_{\text {exc, } \boldsymbol{k}}-\sigma_{\boldsymbol{k}}^{\dagger} \sigma_{\boldsymbol{k}}\right)$ describes the energetics of the manifold of dark exciton states which do not couple to the SP fields and which contains $N_{x} N_{y}\left(N_{z}-1\right)$ exciton states (so that the original number of exciton states is conserved). $\mathbb{I}_{e x c, \boldsymbol{k}}$ is the identity operator on the Hilbert space defined by $\left\{\sigma_{\boldsymbol{k} s}^{\dagger}\right\}_{s=1}^{N_{z}}$. Finally, $H_{u m k l a p p, \boldsymbol{k}}=$ $\sum_{\boldsymbol{q}=\left(\frac{2 \pi q_{x}}{\Delta x}, \frac{2 \pi q_{y}}{\Delta y}\right) \neq 0}\left[\hbar \omega_{\boldsymbol{k}+\boldsymbol{q}}^{S P} a_{\boldsymbol{k}+\boldsymbol{q}}^{\dagger} a_{\boldsymbol{k}+\boldsymbol{q}}+\left(\mathcal{J}_{\boldsymbol{k}+\boldsymbol{q}} \sigma_{\boldsymbol{k}}^{\dagger} a_{\boldsymbol{k}+\boldsymbol{q}}+\right.\right.$ h.c. $\left.)\right]$, where $\boldsymbol{q}$ is a vector of integers, reflects the fact that excitons with a wavevector $\boldsymbol{k}$ can couple to an infinite countable set of SP modes beyond the first Brillouin zone. For our purposes, $H_{u m k l a p p, k}$ can be ignored, as its associated SP modes are far off-resonant with respect to the exciton states.

A straightforward way to generalize this result to an isotropic sample is to assume that each location $r_{\boldsymbol{n}}$ contains three equivalent excitonic transitions with transition dipole amplitudes $\frac{\left|\boldsymbol{\mu}_{e g}\right|}{\sqrt{3}}$ labeled by $\sigma_{\boldsymbol{n} s, \hat{\boldsymbol{j}}}^{\dagger}$ along the $j=x, y, z$ directions. The results above can be recycled provided that we define $\sigma_{\boldsymbol{n} s}^{\dagger}=\frac{\sum_{j}\left(E_{\boldsymbol{k}} \cdot \hat{\boldsymbol{j}}\right) \sigma_{\boldsymbol{n} s, j}^{\dagger}}{\sqrt{\sum_{j}\left|E_{\boldsymbol{k}} \cdot \hat{\boldsymbol{j}}\right|^{2}}}$ and adapt our definition $J_{\boldsymbol{k}}(z)=\sqrt{S}\left(\sqrt{\frac{\hbar \omega_{\boldsymbol{k}}^{S P}}{2 \epsilon_{0} S L_{\boldsymbol{k}}}}\left|\boldsymbol{\mu}_{e g}\right| \frac{\sum_{j} \hat{\boldsymbol{j}}}{\sqrt{3}} \cdot \boldsymbol{E}_{\boldsymbol{k}} e^{-\alpha_{\boldsymbol{k}} z}\right)$ upon which $\mathcal{J}_{\boldsymbol{k}}=\frac{\mathcal{J}_{\boldsymbol{k}}\left(\boldsymbol{E}_{\boldsymbol{k}}=\hat{\boldsymbol{k}}\right)+\mathcal{J}_{\boldsymbol{k}}\left(\boldsymbol{E}_{\boldsymbol{k}}=\frac{i|\boldsymbol{k}|}{\alpha_{d k}} \hat{\boldsymbol{z}}\right)}{3}[58]$.

\section{EVALUATION OF PLEXCITON PL RATE}

\section{A. Fermi's golden rule calculation}

By defining $\boldsymbol{K}=\boldsymbol{K}_{\perp}+K_{z d} \hat{\boldsymbol{z}}$, where $K_{z d}=\boldsymbol{K} \cdot \hat{\boldsymbol{z}} \hat{\boldsymbol{z}}$ and indexing the sums over UHP modes as $\sum_{\boldsymbol{K}, \chi}=\sum_{\boldsymbol{K}_{\perp}, \chi} \sum_{K_{z d}}$, we rewrite Eq. (4) as $\hat{\mathcal{E}}_{U H P}\left(\boldsymbol{r}_{\boldsymbol{n} s}\right)=\hat{\mathcal{E}}_{0}\left(\boldsymbol{r}_{\boldsymbol{n} s}\right)+\hat{\mathcal{E}}_{\text {ref }}\left(\boldsymbol{r}_{\boldsymbol{n} s}\right)$, where

$$
\begin{aligned}
\hat{\mathcal{E}}_{0}(\boldsymbol{r}) & =\sum_{\boldsymbol{K}_{\perp}, \chi} \sum_{K_{z d}} \Theta(-\boldsymbol{K} \cdot \hat{\boldsymbol{z}})\left[\frac{\left(b_{\boldsymbol{K}, \chi}+b_{\boldsymbol{K}_{\perp}+K_{z d} \hat{\boldsymbol{z}}, \chi}\right)}{\sqrt{1+\left|r_{\boldsymbol{K} \chi}\right|^{2}}} \sqrt{\frac{\hbar \omega_{\boldsymbol{K}}^{U H P}}{2 \epsilon_{0} \epsilon_{d} V}} \boldsymbol{e}_{\boldsymbol{K}, \chi} e^{i \boldsymbol{K} \cdot \boldsymbol{r}}\right]+\text { h.c., } \\
\hat{\mathcal{E}}_{r e f}(\boldsymbol{r}) & =\sum_{\boldsymbol{K}_{\perp, \chi}} \sum_{K_{z d}} \Theta(-\boldsymbol{K} \cdot \hat{\boldsymbol{z}})\left[\frac{\left(b_{\boldsymbol{K}, \chi}+b_{\boldsymbol{K}_{\perp}+K_{z d} \hat{\boldsymbol{z}}, \chi}\right) r_{\boldsymbol{K}, \chi}}{\sqrt{1+\left|r_{\boldsymbol{K} \chi}\right|^{2}}} \sqrt{\frac{\hbar \omega_{\boldsymbol{K}}^{U H P}}{2 \epsilon_{0} \epsilon_{d} V}} e^{i \boldsymbol{K}_{\perp} \cdot \boldsymbol{r}-i K_{z d} \boldsymbol{r} \cdot \hat{\boldsymbol{z}}} \boldsymbol{e}_{\boldsymbol{K}_{\perp}+K_{z d} \hat{\boldsymbol{z}}, \chi}\right]+\text { h.c. }
\end{aligned}
$$


The Fermi golden rule expression in Eq. [3] can be naturally partitioned as,

$$
\gamma_{s_{k}}=\gamma_{s_{k}, 0}+\gamma_{s_{k}, r e f}
$$

respectively denoting incoherent contributions independently due to the free-space and reflected waves, and a coherent term between them. Specifically,

$$
\begin{aligned}
\gamma_{y_{\boldsymbol{k}}, 0}=2 \times \frac{2 \pi}{\hbar} \sum_{\boldsymbol{K}_{\perp}, \chi} \sum_{K_{z d}<0}\left[\left|\left\langle\operatorname{vac} ;(\boldsymbol{K}, \chi)_{U H P}\left|-\sum_{\boldsymbol{n} s} \hat{\boldsymbol{\mu}}_{\boldsymbol{n} s} \cdot \hat{\mathcal{E}}_{0}\left(\boldsymbol{r}_{\boldsymbol{n} s}\right)\right| y_{\boldsymbol{k}} ; 0_{U H P}\right\rangle\right|^{2}\right. \\
\left.\quad+\left|\left\langle\operatorname{vac} ;(\boldsymbol{K}, \chi)_{U H P}\left|-\sum_{\boldsymbol{n} s} \hat{\boldsymbol{\mu}}_{\boldsymbol{n} s} \cdot \hat{\mathcal{E}}_{r e f}\left(\boldsymbol{r}_{\boldsymbol{n} s}\right)\right| y_{\boldsymbol{k}} ; 0_{U H P}\right\rangle\right|^{2}\right] \delta\left(\hbar \omega_{y_{\boldsymbol{k}}}-\hbar \omega_{\boldsymbol{K}}^{U H P}\right), \\
\gamma_{y_{\boldsymbol{k}}, r e f}=2 \times \frac{2 \pi}{\hbar} \sum_{\boldsymbol{K}_{\perp}, \chi} \sum_{K_{z d}<0} 2 \Re\left[\left\langle y_{\boldsymbol{k}} ; 0_{U H P}\left|\sum_{\boldsymbol{n} s} \hat{\boldsymbol{\mu}}_{\boldsymbol{n} s} \cdot \hat{\mathcal{E}}_{r e f}\left(\boldsymbol{r}_{\boldsymbol{n} s}\right)\right| \operatorname{vac} ;(\boldsymbol{K}, \chi)_{U H P}\right\rangle\right. \\
\left.\quad \times\left\langle\operatorname{vac} ;(\boldsymbol{K}, \chi)_{U H P}\left|\sum_{\boldsymbol{n}^{\prime} s^{\prime}} \hat{\boldsymbol{\mu}}_{\boldsymbol{n}^{\prime} s^{\prime}} \cdot \hat{\mathcal{E}}_{0}\left(\boldsymbol{r}_{\boldsymbol{n}^{\prime} s^{\prime}}\right)\right| y_{\boldsymbol{k}} ; 0_{U H P}\right\rangle\right] \delta\left(\hbar \omega_{y_{\boldsymbol{k}}}-\hbar \omega_{\boldsymbol{K}}^{U H P}\right)
\end{aligned}
$$

where we have recognized the symmetry $\sum_{K_{z d}}=2 \sum_{K_{z d}<0}$. To evaluate the expressions above, the following matrix element for $K_{z d}<0$ is handy,

$$
\begin{aligned}
& \left\langle\operatorname{vac} ;(\boldsymbol{K}, \chi)_{U H P}\left|-\sum_{\boldsymbol{n} s} \hat{\boldsymbol{\mu}}_{\boldsymbol{n} s} \cdot \hat{\mathcal{E}}_{0}\left(\boldsymbol{r}_{\boldsymbol{n} s}\right)\right| y_{\boldsymbol{k}} ; 0_{U H P}\right\rangle \\
& =-\zeta_{y_{\boldsymbol{k}}}^{(e x c)}\left\langle\operatorname{vac} ;(\boldsymbol{K}, \chi)_{U H P}\left|\sum_{\boldsymbol{n} s} \hat{\boldsymbol{\mu}}_{\boldsymbol{n} s} \cdot \hat{\mathcal{E}}_{0}\left(\boldsymbol{r}_{\boldsymbol{n} s}\right) d_{\boldsymbol{n} s} \sigma_{\boldsymbol{n} s}^{\dagger}\right| \operatorname{vac} ; 0_{U H P}\right\rangle \\
& =-\frac{\zeta_{y_{\boldsymbol{k}}}^{(e x c)}}{\sqrt{1+\left|r_{\boldsymbol{K} \chi}\right|^{2}}} \sqrt{\frac{\hbar \omega_{\boldsymbol{K}}^{U H P}}{2 \epsilon_{0} \epsilon_{d} V}} \boldsymbol{\mu}_{g e} \cdot \boldsymbol{e}_{\boldsymbol{K}, \chi}^{*} \sum_{\boldsymbol{n} s} e^{-i \boldsymbol{K}_{\perp} \cdot \boldsymbol{r}_{n}} e^{-i K_{z d} z_{s}} \sqrt{\frac{1}{S}} \frac{J_{\boldsymbol{k}}\left(z_{s}\right)}{\mathcal{J}_{\boldsymbol{k}}} e^{i \boldsymbol{k} \cdot \boldsymbol{r}_{\boldsymbol{n}}} \\
& =-\frac{\zeta_{y_{\boldsymbol{k}}}^{(e x c)}}{\sqrt{1+\left|r_{\boldsymbol{K} \chi}\right|^{2}}} \sqrt{\frac{\hbar \omega_{\boldsymbol{K}}^{U H P}}{2 \epsilon_{0} \epsilon_{d} V}} \boldsymbol{\mu}_{g e} \cdot \boldsymbol{e}_{\boldsymbol{K}, \chi}^{*} \sum_{\boldsymbol{n} s} e^{-i \boldsymbol{K}_{\perp} \cdot \boldsymbol{r}_{\boldsymbol{n}}} e^{-i K_{z d} z_{s}} \sqrt{\frac{1}{S}} \frac{J_{\boldsymbol{k}}\left(z_{s}\right)}{\mathcal{J}_{\boldsymbol{k}}} e^{i \boldsymbol{k} \cdot \boldsymbol{r}_{\boldsymbol{n}}} \\
& =-\frac{\zeta_{y_{\boldsymbol{k}}}^{(e x c)}}{\sqrt{1+\left|r_{\boldsymbol{K} \chi}\right|^{2}}} \sqrt{\frac{\hbar \omega_{\boldsymbol{K}}^{U H P}}{2 \epsilon_{0} \epsilon_{d} V}} \boldsymbol{\mu}_{g e} \cdot \boldsymbol{e}_{\boldsymbol{K}, \chi}^{*} N_{x} N_{y} \delta_{\boldsymbol{K}_{\perp}, \boldsymbol{k}} \sqrt{\frac{1}{S}} \frac{\frac{N_{z}}{W_{z}} \int_{z_{0}}^{z_{f}} d z J_{\boldsymbol{k}}(z) e^{-i K_{z d} z}}{\sqrt{\rho \int_{z_{0}}^{z_{f}} d z\left|J_{\boldsymbol{k}}(z)\right|^{2}}},
\end{aligned}
$$

where we have approximated the sum over layers by an integral $\sum_{s} \rightarrow \frac{N_{z}}{W_{z}} \int_{z_{0}}^{z_{f}} d z$. Proceeding similarly with the reflected waves, we have for $K_{d z}<0$,

$$
\begin{aligned}
& \left\langle\operatorname{vac} ;(\boldsymbol{K}, \chi)_{U H P}\left|-\sum_{\boldsymbol{n} s} \hat{\boldsymbol{\mu}}_{\boldsymbol{n} s} \cdot \hat{\mathcal{E}}_{r e f}\left(\boldsymbol{r}_{\boldsymbol{n} s}\right)\right| y_{\boldsymbol{k}} ; 0_{U H P}\right\rangle \\
= & -\frac{r_{\boldsymbol{K} \chi}^{*} \zeta_{y_{\boldsymbol{k}}}^{(e x c)}}{\sqrt{1+\left|r_{\boldsymbol{K} \chi}\right|^{2}}} \sqrt{\frac{\hbar \omega_{\boldsymbol{K}}^{U H P}}{2 \epsilon_{0} \epsilon_{d} V}} \boldsymbol{\mu}_{g e} \cdot \boldsymbol{e}_{\boldsymbol{K}_{\perp}-K_{z d} \hat{\boldsymbol{z}}, \chi}^{*} N_{x} N_{y} \delta_{\boldsymbol{K}_{\perp}, \boldsymbol{k}} \sqrt{\frac{1}{S}} \frac{\frac{N_{z}}{W_{z}} \int_{z_{0}}^{z_{f}} d z J_{\boldsymbol{k}}(z) e^{i K_{z d} z}}{\sqrt{\rho \int_{z_{0}}^{z_{f}} d z\left|J_{\boldsymbol{k}}(z)\right|^{2}}}
\end{aligned}
$$

The quasi-momentum conservation $\delta_{\boldsymbol{K}_{\perp}, \boldsymbol{k}}$ terms in Eqs. S20 and S21) reveal that a plexciton with in-plane wavevector $\boldsymbol{k}$ will emit UHP photons with the same in-plane wavevector.

To begin our evaluation of the rates, we plug Eq. S20 into Eq. S19a and simplify the resulting expression, 


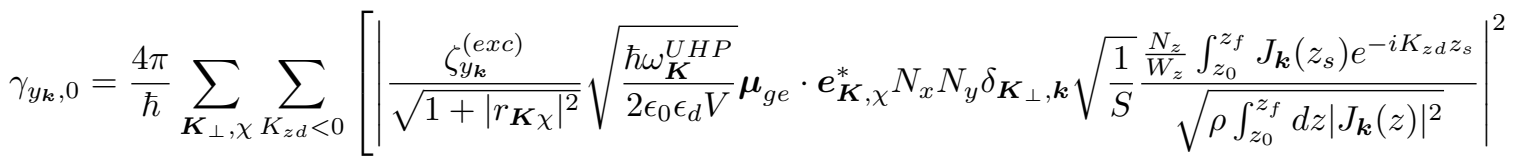

$$
\begin{aligned}
& \left.+\left|\frac{r_{\boldsymbol{K}_{\chi}}^{*} \zeta_{y_{\boldsymbol{k}}}^{(e x c)}}{\sqrt{1+\left|r_{\boldsymbol{K} \chi}\right|^{2}}} \sqrt{\frac{\hbar \omega_{\boldsymbol{K}}^{U H P}}{2 \epsilon_{0} \epsilon_{d} V}} \boldsymbol{\mu}_{g e} \cdot \boldsymbol{e}_{\boldsymbol{K}_{\perp}-K_{z d} \hat{\boldsymbol{z}}, \chi}^{*} N_{x} N_{y} \delta_{\boldsymbol{K}_{\perp}, \boldsymbol{k}} \sqrt{\frac{1}{S}} \frac{\frac{N_{z}}{W_{z}} \int_{z_{0}}^{z_{f}} J_{\boldsymbol{k}}\left(z_{s}\right) e^{i K_{z d} z_{s}}}{\sqrt{\rho \int_{z_{0}}^{z_{f}} d z\left|J_{\boldsymbol{k}}(z)\right|^{2}}}\right|^{2}\right] \delta\left(\hbar \omega_{y_{\boldsymbol{k}}}-\hbar \omega_{\boldsymbol{K}}^{U H P}\right) \\
& =\frac{\left|\zeta_{y_{\boldsymbol{k}}}^{(e x c)}\right|^{2}}{\left(1+\left|r_{\boldsymbol{K} \chi}\right|^{2}\right)} \frac{4 \pi}{\hbar}\left(\frac{L_{z}}{2 \pi} \int_{-\infty}^{0} d K_{z d}\right) \sum_{q= \pm} \sum_{\chi} \frac{\hbar \omega_{\boldsymbol{k}, s}}{2 \epsilon_{0} \epsilon_{d} V}\left|\boldsymbol{\mu}_{e g} \cdot \boldsymbol{e}_{\boldsymbol{K}, \chi}\right|^{2} \\
& \times \frac{N_{x}^{2} N_{y}^{2}}{S}\left(\frac{N_{z}}{W_{z}}\right)^{2}\left[\frac{\left|\int_{z_{0}}^{z_{f}} d z J_{\boldsymbol{k}}(z) e^{-i K_{z d} z}\right|^{2}+\left|r_{\boldsymbol{K} \chi}\right|^{2}\left|\int_{z_{0}}^{z_{f}} d z J_{\boldsymbol{k}}(z) e^{i K_{z d} z}\right|^{2}}{\rho \int_{z_{0}}^{z_{f}} d z\left|J_{\boldsymbol{k}}(z)\right|^{2}}\right] \\
& \times \Theta\left(\omega_{\boldsymbol{k}, y}-\frac{c}{\sqrt{\epsilon_{d}}}|\boldsymbol{k}|\right) \frac{\sqrt{|\boldsymbol{k}|^{2}+k_{d z}^{2}} \delta\left(K_{z d}+k_{d z}\right)}{\frac{\hbar c}{\sqrt{\epsilon_{d}}} k_{d z}} \\
& =\left|\zeta_{y_{\boldsymbol{k}}}^{(e x c)}\right|^{2}\left(\frac{\rho \omega_{\boldsymbol{k}, y}^{2}}{2 \epsilon_{0} \hbar c^{2}}\right) \Theta\left(\omega_{\boldsymbol{k}, y}-\frac{c}{\sqrt{\epsilon_{d}}}|\boldsymbol{k}|\right) \frac{\sum_{q= \pm} \frac{1}{k_{d z}} \sum_{\chi}\left|\boldsymbol{\mu}_{e g} \cdot \boldsymbol{e}_{\boldsymbol{k}+q k_{d z} \hat{\boldsymbol{z}}, \chi}\right|^{2}\left|\int_{z_{0}}^{z_{f}} d z J_{\boldsymbol{k}}(z) e^{-i q k_{z d} z}\right|^{2}}{\int_{z_{0}}^{z_{f}} d z\left|J_{\boldsymbol{k}}(z)\right|^{2}} .
\end{aligned}
$$

To go from the first to the second equality, we converted $\sum_{K_{z d}<0} \rightarrow \frac{L_{z}}{2 \pi} \int_{-\infty}^{0} d K_{z d}$, where $V=S L_{z}$ is the UHP quantization volume. Note that while we considered the quantization area to be equal to the plexciton setup surface area $S$, the quantization box vertical dimension $L_{z}$ is not equal to the thickness of the organic layer $W_{z}$ in general. We have also used the $\delta_{\boldsymbol{K}_{\perp}, \boldsymbol{k}}$ condition to rewrite the energy-conservation restriction as a constraint on the vertical momentum of the emited photon $\delta\left(\hbar \omega_{\boldsymbol{k}, y}-\hbar \omega_{\boldsymbol{K}}^{U H P}\right)=\Theta\left(\omega_{\boldsymbol{k}, s}-\frac{c}{\sqrt{\epsilon_{d}}}|\boldsymbol{k}|\right) \frac{\sqrt{|\boldsymbol{k}|^{2}+k_{d z}^{2}}\left[\delta\left(K_{z d}-k_{z d}\right)+\delta\left(K_{z d}+k_{z d}\right)\right]}{\frac{\hbar c}{\sqrt{\epsilon_{d}} k_{d z}}}$, where we have used the shorthand notation

$$
k_{z d}=\sqrt{\frac{\epsilon_{d} \omega_{\boldsymbol{k}, y}^{2}}{c^{2}}-|\boldsymbol{k}|^{2}}>0 .
$$

Finally, we used the fact that for a lossless metal $(\Gamma=0)$ and for $\omega_{K}^{U H P}<\frac{\omega_{P}}{\sqrt{\epsilon_{d}+\epsilon_{\infty}}}$ (the UP frequency is below the asymptotic SP frequency for $k \rightarrow \infty$, which is typically the case), $K_{z d} \in \Re$ and $K_{z m} \in \Im$, implying that $\left|r_{K_{\chi}}\right|^{2}=1$, i.e., metals are perfectly reflective. Proceeding analogously by plugging Eqs. (S20) and (S21) into Eq. (S19b), we obtain,

$$
\begin{aligned}
\gamma_{y_{\boldsymbol{k}}, r e f}= & \left|\zeta_{y_{\boldsymbol{k}}}^{(e x c)}\right|^{2}\left(\frac{\rho \omega_{\boldsymbol{k}, y}^{2}}{2 \epsilon_{0} \hbar c^{2}}\right) \Theta\left(\omega_{\boldsymbol{k}, y}-\frac{c}{\sqrt{\epsilon_{d}}}|\boldsymbol{k}|\right) \\
& \times \frac{\Re\left[2 \sum_{\chi} \frac{1}{k_{d z}} r_{\boldsymbol{k}-k_{d z} \hat{\boldsymbol{z}}, \chi}\left(\boldsymbol{e}_{\boldsymbol{k}+k_{d z} \hat{\boldsymbol{z}}, \chi} \cdot \boldsymbol{\mu}_{e g}\right)\left(\boldsymbol{\mu}_{g e} \cdot \boldsymbol{e}_{\boldsymbol{k}-k_{d z} \hat{\boldsymbol{z}}, \chi}^{*}\right) \int_{z_{0}}^{z_{f}} d z J_{\boldsymbol{k}}^{*}(z) e^{i k_{z d} z} \int_{z_{0}}^{z_{f}} d z^{\prime} J_{\boldsymbol{k}}\left(z^{\prime}\right) e^{i k_{z d} z^{\prime}}\right]}{\int_{z_{0}}^{z_{f}} d z\left|J_{\boldsymbol{k}}(z)\right|^{2}}
\end{aligned}
$$

\section{B. Connection with Green's function formalism}

\section{Dyadic Green's function}

The quantum dyadic Green's function $\overleftrightarrow{G}$ is the half-sided Fourier transform of the correlation function of the electric field in the region of interest, $\hat{\mathcal{E}}_{U H P}(\boldsymbol{r}, t)=\sum_{\mu} \sqrt{\frac{\hbar \omega_{\mu}}{2 \epsilon_{0}}} b_{\mu}(t) \boldsymbol{F}_{\mu}(\boldsymbol{r})+$ h.c. [59, 60, 


$$
\begin{aligned}
G\left(\boldsymbol{r}, \boldsymbol{r}^{\prime}, \omega\right) & =\frac{i c^{2} \epsilon_{0}}{\hbar \omega^{2}} \int_{0}^{\infty} d t e^{i \omega t}\left\langle\left[\hat{\mathcal{E}}_{U H P}(\boldsymbol{r}, t), \hat{\mathcal{E}}_{U H P}\left(\boldsymbol{r}^{\prime}, 0\right)\right]\right\rangle \\
& =\lim _{\epsilon \rightarrow 0^{+}} \sum_{\eta} c^{2} \frac{\boldsymbol{F}_{\eta}(\boldsymbol{r}) \otimes \boldsymbol{F}_{\eta}^{*}\left(\boldsymbol{r}^{\prime}\right)}{\omega_{\eta}^{2}-(\omega+i \epsilon)^{2}}
\end{aligned}
$$

where $\boldsymbol{F}_{\mu}(\boldsymbol{r})$ is the electric field profile of the $\eta$ th mode satisfying the orthonormality relation $\int d^{3} r \epsilon(\boldsymbol{r}) \boldsymbol{F}_{\eta}(\boldsymbol{r}) \boldsymbol{F}_{\eta^{\prime}}^{*}(\boldsymbol{r})=$ $\delta_{\eta \eta^{\prime}}$. From Eq. $[\mathrm{S} 26]$ it follows that [31]

$$
\Im G_{\beta \alpha}\left(\boldsymbol{r}, \boldsymbol{r}^{\prime}, \omega\right)=\frac{\pi c^{2}}{2 \omega} \sum_{\eta}\left[\boldsymbol{F}_{\eta}(\boldsymbol{r})\right]_{\beta}\left[\boldsymbol{F}_{\eta}^{*}\left(\boldsymbol{r}^{\prime}\right)\right]_{\alpha} \delta\left(\omega_{\mu}-\omega\right),
$$

where we have ignored the negative frequency poles. Fermi's golden rule rate in Eq. (3) can be readily expressed in terms of $G_{\beta \alpha}$ using Eq. S27,

$$
\begin{aligned}
\gamma_{y_{\boldsymbol{k}}}^{\text {Green }} & =\frac{2 \pi}{\hbar} \sum_{\eta} \mid\left.\left\langle\text { vac; } \eta\left|-\sum_{\boldsymbol{n} s} \hat{\boldsymbol{\mu}}_{\boldsymbol{n} s} \cdot \hat{\mathcal{E}}_{U H P}\left(\boldsymbol{r}_{\boldsymbol{n} s}\right)\right| y_{\boldsymbol{k}} ; 0_{U H P}\right\rangle\right|^{2} \delta\left(\hbar \omega_{\eta}-\hbar \omega_{y_{\boldsymbol{k}}}\right) \\
& =\frac{2 \pi}{\hbar}\left|\zeta_{y_{\boldsymbol{k}}}^{(e x c)}\right|^{2} \sum_{\eta} \sum_{\boldsymbol{n} s ; \boldsymbol{n}^{\prime} s^{\prime}} \frac{\hbar \omega_{\mu}}{2 \epsilon_{0}} d_{\boldsymbol{n} s}^{(\boldsymbol{k}) *} \sum_{\alpha, \beta} \mu_{e g}^{\beta}\left[\boldsymbol{F}_{\eta}\left(\boldsymbol{r}_{\boldsymbol{n} s}\right)\right]_{\beta}\left[\boldsymbol{F}_{\eta}^{*}\left(\boldsymbol{r}_{\boldsymbol{n}^{\prime} s^{\prime}}\right)\right]_{\alpha} \mu_{g e}^{\alpha} d_{\boldsymbol{n}^{\prime} s^{\prime}}^{(\boldsymbol{k})} \delta\left(\hbar \omega_{\eta}-\hbar \omega_{y_{\boldsymbol{k}}}\right) \\
& =\left|\zeta_{y_{\boldsymbol{k}}}^{(e x c)}\right|^{2}\left(\frac{2 \omega_{\boldsymbol{k}, y}^{2}}{\epsilon_{0} \hbar c^{2}}\right) \Im \sum_{\boldsymbol{n} \boldsymbol{s} ; \boldsymbol{n}^{\prime} s^{\prime}} d_{\boldsymbol{n} s}^{(\boldsymbol{k}) *}\left[\sum_{\alpha, \beta} \mu_{e g}^{\beta} G_{\beta \alpha}\left(\boldsymbol{r}_{\boldsymbol{n} s}, \boldsymbol{r}_{\boldsymbol{n}^{\prime} s^{\prime}}, \omega_{\boldsymbol{k}, s}\right) \mu_{g e}^{\alpha}\right] d_{\boldsymbol{n}^{\prime} s^{\prime}}^{(\boldsymbol{k})}
\end{aligned}
$$

Owing to the harmonic nature of electromagnetic excitations, the quantum dyadic Green's function in Eq. (S26) is equivalent to its classical counterpart [59, 60]. The textbook expression for the classical dyadic corresponding to our problem is [31, 38, 39],

$$
\overleftrightarrow{G}_{t e x t}=\overleftrightarrow{G}_{0}\left(\boldsymbol{r}_{\boldsymbol{n} s}, \boldsymbol{r}_{\boldsymbol{n}^{\prime} s^{\prime}}, \omega\right)+\overleftrightarrow{G}_{r e f}\left(\boldsymbol{r}_{\boldsymbol{n} s}, \boldsymbol{r}_{\boldsymbol{n}^{\prime} s^{\prime}}, \omega\right)
$$

As we will next show, inserting Eq. (S29) into Eq. (S28) gives rise to identical expressions to Eq. (S23) and (S25a), representing direct and interference terms involving free-space and reflected waves, respectively.

$\overleftrightarrow{G_{0}}$ corresponds to the zeroth order free-space propagation of the electromagnetic field in the absence of the metal surface,

$$
\overleftrightarrow{G}_{0}\left(\boldsymbol{r}_{\boldsymbol{n} s}, \boldsymbol{r}_{\boldsymbol{n}^{\prime} s^{\prime}}, \omega\right)=\frac{i}{8 \pi^{2}} \int_{-\infty}^{\infty} d K_{x} \int_{-\infty}^{\infty} d K_{y} e^{\left.i\left[\boldsymbol{K}_{\perp} \cdot\left(\boldsymbol{r}_{\boldsymbol{n}}-\boldsymbol{r}_{\boldsymbol{n}^{\prime}}\right)+k_{z d} \mid z_{s}-z_{s^{\prime}}\right]\right]} \overleftrightarrow{M}_{0}\left(\omega, \boldsymbol{K}_{\perp}, z_{s}, z_{s^{\prime}}\right)
$$

where $\overleftrightarrow{M}_{0}\left(\omega, \boldsymbol{K}_{\perp}, z_{s}, z_{s^{\prime}}\right)=\overleftrightarrow{M}_{0 \pm}\left(\omega, \boldsymbol{K}_{\perp}\right)=\overleftrightarrow{M}_{0 \pm}^{s}\left(\omega, \boldsymbol{K}_{\perp}\right)+\overleftrightarrow{M}_{0 \pm}^{p}\left(\omega, \boldsymbol{K}_{\perp}\right)$. Here, the positive sign is chosen if $z_{s}>z_{s^{\prime}}$ and the negative one if $z_{s}<z_{s^{\prime}}$; the average $\overleftrightarrow{M}_{0}\left(\omega, \boldsymbol{K}_{\perp}, z_{s}, z_{s^{\prime}}\right)=\frac{1}{2}\left[\overleftrightarrow{M}_{0+}\left(\omega, \boldsymbol{K}_{\perp}\right)+\overleftrightarrow{M}_{0-}\left(\omega, \boldsymbol{K}_{\perp}\right)\right]$ is evaluated if $z_{s}=z_{s^{\prime}}$. These matrices can be written as outer products of the free-space photon polarizations, $\overleftrightarrow{M}_{0 q, \beta \alpha}^{\chi}\left(\omega, \boldsymbol{K}_{\perp}\right)=$ $\frac{1}{k_{z d}}\left(\boldsymbol{e}_{\boldsymbol{K}_{\perp}+q k_{z d} \hat{\boldsymbol{z}}, \chi}\right)_{\beta}\left(\boldsymbol{e}_{\boldsymbol{K}_{\perp}+q k_{z d} \hat{\boldsymbol{z}}, \chi}\right)_{\alpha}^{*}[38$. More explicitly [31],

$$
\overleftrightarrow{M}_{0 \pm}=\frac{1}{K_{d}^{2} k_{z d}}\left[\begin{array}{ccc}
K_{d}^{2}-K_{x}^{2} & -K_{x} K_{y} & \mp K_{x} k_{z d} \\
-K_{x} K_{y} & K_{d}^{2}-K_{y}^{2} & \mp K_{y} k_{z d} \\
\mp K_{x} k_{z d} & \mp K_{y} k_{z d} & K_{d}^{2}-k_{z d}^{2}
\end{array}\right]
$$

We have used the notation $K_{d}(\omega)=|\boldsymbol{K}(\omega)|=\frac{\sqrt{\epsilon_{d}} \omega}{c}$. On the other hand, $\overleftrightarrow{G}_{\text {ref }}$ corresponds to the reflected electromagnetic waves from the metal-dielectric interface,

$$
\overleftrightarrow{G}_{r e f}\left(\boldsymbol{r}_{\boldsymbol{n} s}, \boldsymbol{r}_{\boldsymbol{n}^{\prime} s^{\prime}}, \omega\right)=\frac{i}{8 \pi^{2}} \int_{|\boldsymbol{K}|<\frac{\sqrt{\epsilon_{d}} \omega}{c}} d^{2} \boldsymbol{K} e^{i\left[\boldsymbol{K} \cdot\left(\boldsymbol{r}_{n}-\boldsymbol{r}_{n^{\prime}}\right)+k_{z d}\left(z_{s}+z_{s^{\prime}}\right)\right]} \overleftrightarrow{M}_{r e f}(\omega, \boldsymbol{k})
$$


with $\overleftrightarrow{M}_{r e f}(\omega, \boldsymbol{k})=\overleftrightarrow{M}_{r e f}^{s}(\omega, \boldsymbol{k})+\overleftrightarrow{M}_{r e f}^{p}(\omega, \boldsymbol{k})$, where $\overleftrightarrow{M}_{r e f, \beta \alpha}^{\chi}\left(\omega, \boldsymbol{K}_{\perp}\right)=\frac{r_{K, \chi}}{K_{z d}}\left(\boldsymbol{e}_{\boldsymbol{K}_{\perp}+k_{z d} \hat{\boldsymbol{z}}, \chi}\right)_{\beta}\left(\boldsymbol{e}_{\boldsymbol{K}_{\perp}-k_{z d} \hat{\boldsymbol{z}}, \chi}\right)_{\alpha}^{*}[38]$, where 31

$$
\begin{aligned}
\overleftrightarrow{M}_{r e f}^{s}(\omega, \boldsymbol{K}) & =\frac{r_{\boldsymbol{K}, s}}{k_{z d}\left(K_{x}^{2}+K_{y}^{2}\right)}\left[\begin{array}{ccc}
K_{y}^{2} & -K_{x} K_{y} & 0 \\
-K_{x} K_{y} & K_{x}^{2} & 0 \\
0 & 0 & 0
\end{array}\right] \\
\overleftrightarrow{M}_{r e f}^{p}(\omega, \boldsymbol{k}) & =\frac{-r_{\boldsymbol{K}, p}}{K_{d}^{2}\left(K_{x}^{2}+K_{y}^{2}\right)}\left[\begin{array}{ccc}
K_{x}^{2} k_{z d} & K_{x} K_{y} k_{z d} & K_{x}\left(K_{x}^{2}+K_{y}^{2}\right) \\
K_{x} K_{y} k_{z d} & K_{y}^{2} k_{z d} & K_{y}\left(K_{x}^{2}+K_{y}^{2}\right) \\
-K_{x}\left(K_{x}^{2}+K_{y}^{2}\right) & -K_{y}\left(K_{x}^{2}+K_{y}^{2}\right) & -\left(K_{x}^{2}+K_{y}^{2}\right)^{2} / k_{z d}
\end{array}\right]
\end{aligned}
$$

Notice that we have limited Eq. $\sqrt{\mathrm{S} 32}$ to the integration $\int_{|\boldsymbol{K}|<\frac{\sqrt{\epsilon_{d}} \omega}{c}} d^{2} \boldsymbol{K}$ over radiative modes. For real valued relative permittivities, $\epsilon_{d}$ and $\epsilon_{m}$, integration over evanescent wavevectors (with $K_{d z} \in \Im$ ) picks up the corresponding SP mode pole contributions. We need not take these into account, since we have already accounted for SP modes in our strong-coupling Hamiltonian formalism.

\section{Evaluation of Eq. S28}

We now proceed to directly evaluate Eq. $(\mathrm{S} 28)$ for the different terms of $\overleftrightarrow{G}_{\text {text }}$ in Eq. S29. Starting with the free-space term,

$$
\begin{aligned}
& \gamma_{y_{\boldsymbol{k}}, 0}^{\text {Green }} \\
& =\left|\zeta_{y_{\boldsymbol{k}}}^{(e x c)}\right|^{2}\left(\frac{2 \omega_{\boldsymbol{k}, y}^{2}}{\epsilon_{0} \hbar c^{2}}\right) \Im \sum_{\boldsymbol{n} s ; \boldsymbol{n}^{\prime} s^{\prime}} d_{\boldsymbol{n} s}^{(\boldsymbol{k}) *} d_{\boldsymbol{n}^{\prime} s^{\prime}}^{(\boldsymbol{k})}\left[\sum_{\alpha, \beta} \mu_{g e}^{\beta} G_{0, \beta \alpha}\left(\boldsymbol{r}_{\boldsymbol{n} s}, \boldsymbol{r}_{\boldsymbol{n}^{\prime} s^{\prime}}, \omega_{\boldsymbol{k}, s}\right) \mu_{e g}^{\alpha}\right] \\
& =\left|\zeta_{y_{\boldsymbol{k}}}^{(e x c)}\right|^{2}\left(\frac{2 \omega_{\boldsymbol{k}, y}^{2}}{\epsilon_{0} \hbar c^{2}}\right) \Im \sum_{\boldsymbol{n} s ; \boldsymbol{n}^{\prime} s^{\prime}} \frac{1}{S} \frac{J_{\boldsymbol{k}}^{*}\left(z_{s}\right) e^{-i \boldsymbol{k} \cdot \boldsymbol{r}_{\boldsymbol{n}}} J_{\boldsymbol{k}}\left(z_{s^{\prime}}\right) e^{i \boldsymbol{k} \cdot \boldsymbol{r}_{n^{\prime}}}}{\mathcal{J}_{\boldsymbol{k}}^{2}} \\
& \times\left\{\frac{i}{8 \pi^{2}} \int_{-\infty}^{\infty} d K_{x} \int_{-\infty}^{\infty} d K_{y} e^{i\left[\boldsymbol{K} \cdot\left(\boldsymbol{r}_{n}-\boldsymbol{r}_{n^{\prime}}\right)+K_{d z}\left|z_{s}-z_{s^{\prime}}\right|\right]} \sum_{\alpha, \beta} \mu_{g e}^{\beta} M_{0, \beta \alpha}\left(\omega_{\boldsymbol{k}, s}, \tilde{\boldsymbol{k}}, z_{s}, z_{s^{\prime}}\right) \mu_{e g}^{\alpha}\right\} \\
& =\left|\zeta_{y_{\boldsymbol{k}}}^{(e x c)}\right|^{2}\left(\frac{2 \omega_{\boldsymbol{k}, y}^{2}}{\epsilon_{0} \hbar c^{2}}\right) \Im \sum_{s s^{\prime}} \frac{1}{S} \frac{J_{\boldsymbol{k}}^{*}\left(z_{s}\right) J_{\boldsymbol{k}}\left(z_{s^{\prime}}\right)}{\mathcal{J}_{\boldsymbol{k}}^{2}}\left[\frac{i}{8 \pi^{2}} \int_{-\infty}^{\infty} d K_{x} \int_{-\infty}^{\infty} d K_{y} N_{x}^{2} N_{y}^{2} \delta_{\boldsymbol{k}, \boldsymbol{K}} e^{i K_{d z}\left|z_{s}-z_{s^{\prime}}\right|} \sum_{\alpha, \beta} \mu_{g e}^{\beta} M_{0, \beta \alpha}\left(\omega_{\boldsymbol{k}, s}, \tilde{\boldsymbol{k}}_{,} z_{s}, z_{s^{\prime}}\right) \mu_{e g}^{\alpha}\right] \\
& =\left|\zeta_{y_{\boldsymbol{k}}}^{(e x c)}\right|^{2}\left(\frac{2 \omega_{\boldsymbol{k}, y}^{2}}{\epsilon_{0} \hbar c^{2}}\right) \Im \sum_{s s^{\prime}} \frac{1}{S} \frac{J_{\boldsymbol{k}}^{*}\left(z_{s}\right) J_{\boldsymbol{k}}\left(z_{s^{\prime}}\right) N_{x}^{2} N_{y}^{2}}{\mathcal{J}_{\boldsymbol{k}}^{2}} \\
& \times\left[\frac{i}{8 \pi^{2}} \int_{-\infty}^{\infty} d K_{x} \int_{-\infty}^{\infty} d K_{y} \frac{(2 \pi)^{2}}{S} \delta(\boldsymbol{k}-\boldsymbol{K}) e^{i k_{d z}\left|z_{s}-z_{s^{\prime}}\right|} \sum_{\alpha, \beta} \mu_{g e}^{\beta} M_{0, \beta \alpha}\left(\omega_{\boldsymbol{k}, s}, \tilde{\boldsymbol{k}}, z_{s}, z_{s^{\prime}}\right) \mu_{e g}^{\alpha}\right] \\
& =\left|\zeta_{y_{\boldsymbol{k}}}^{(e x c)}\right|^{2}\left(\frac{\omega_{\boldsymbol{k}, y}^{2}}{2 \epsilon_{0} \hbar c^{2}}\right) \frac{2 \Re\left\{\left[\sum_{\alpha, \beta} \mu_{g e^{\beta}}^{\beta} M_{0, \beta \alpha}\left(\omega_{\boldsymbol{k}, s}, \boldsymbol{k}, z_{s}, z_{s^{\prime}}\right) \mu_{e g}^{\alpha}\right] \sum_{s s^{\prime}} J_{\boldsymbol{k}}^{*}\left(z_{s}\right) J_{\boldsymbol{k}}\left(z_{s^{\prime}}\right) e^{i k_{d z}\left|z_{s}-z_{s^{\prime}}\right|}\right\}}{\mathcal{J}_{\boldsymbol{k}}^{2}}\left(\frac{N_{x}^{2} N_{y}^{2}}{S^{2}}\right) .
\end{aligned}
$$

Going from the third to the fourth line, we have approximated $\delta_{\boldsymbol{k}, \boldsymbol{K}}=\frac{(2 \pi)^{2}}{S} \delta(\boldsymbol{k}-\boldsymbol{K})$. At a first glance, it is not clear that Eq. S35) is equal to Eq. (S23). However, with some algebraic effort, it is possible to establish the following 
identity,

$$
\begin{aligned}
& 2 \Re\left[\left(\sum_{\alpha, \beta} \mu_{g e}^{\beta} M_{0, \beta \alpha}\left(\omega_{\boldsymbol{k}, y}, \boldsymbol{k}, z_{s}, z_{s^{\prime}}\right) \mu_{e g}^{\alpha}\right) \sum_{s, s^{\prime}} J_{\boldsymbol{k}}^{*}\left(z_{s}\right) J_{\boldsymbol{k}}\left(z_{s^{\prime}}\right) e^{i k_{d z}\left|z_{s}-z_{s^{\prime}}\right|}\right] \\
= & \Theta\left(\omega_{\boldsymbol{k}, y}-\frac{c}{\sqrt{\epsilon_{d}}}|\boldsymbol{k}|\right) \sum_{q= \pm}\left[\sum_{\alpha, \beta} \mu_{g e}^{\beta} M_{0 q, \beta \alpha}\left(\omega_{\boldsymbol{k}, s}, \boldsymbol{k}\right) \mu_{e g}^{\alpha}\left|\sum_{s} J_{\boldsymbol{k}}\left(z_{s}\right) e^{-i q k_{d z} z}\right|^{2}\right] \\
= & \Theta\left(\omega_{\boldsymbol{k}, y}-\frac{c}{\sqrt{\epsilon_{d}}}|\boldsymbol{k}|\right) \sum_{q= \pm}\left[\frac{1}{k_{d z}} \sum_{\chi}\left|\boldsymbol{\mu}_{e g} \cdot \boldsymbol{e}_{\boldsymbol{k}+q k_{d z} \hat{\boldsymbol{z}}, \chi}\right|^{2}\left|\sum_{s} J_{\boldsymbol{k}}\left(z_{s}\right) e^{-i q k_{d z} z}\right|^{2}\right],
\end{aligned}
$$

where we have used the definition of $M_{0 q, \beta \alpha}\left(\omega_{\boldsymbol{k}, y}, \boldsymbol{k}\right)$ presented above. Substituting Eq. (S36) together with $\sum_{s} \rightarrow$ $\frac{N_{z}}{W_{z}} \int_{z_{0}}^{z_{f}} d z$ on Eq. S35 yields $\gamma_{y_{k}, 0}^{G r e e n}=\gamma_{y_{k}, 0}$, thus verifying Eq. S23. Interestingly, the physical interpretations of these two equivalent calculations is slightly different: $\gamma_{y_{k}, 0}$ is the sum of incoherent contributions from free and reflected waves (see Eq. (S19a)) while $\gamma_{y_{k}, 0}^{\text {Green }}$ uses information of free waves alone (see Eqs. (S30) and (S31)). These two physical pictures can be reconciled by noting the form of the modal decomposition of $\hat{\mathcal{E}}_{U H P}$ in Eq. (4). Using similar manipulations, it follows straightforwardly that $\gamma_{y_{\boldsymbol{k}}, \text { ref }}^{\text {Green }}=\gamma_{y_{\boldsymbol{k}}, \text { ref }}$ as in Eq. S25a. We thus obtain $\gamma_{y_{\boldsymbol{k}}}^{\mathrm{Green}}=\gamma_{y_{\boldsymbol{k}}}$.

\section{Final expressions}

We aim to provide simplified expressions that specialize to a common experimental situation where molecules are randomly oriented in the organic layer. Denoting isotropic averages $\langle\cdot\rangle_{i s o}$, the following identities will be useful,

$$
\begin{aligned}
& \left\langle\sum_{q= \pm} \frac{1}{k_{d z}} \sum_{\chi}\left|\boldsymbol{\mu}_{e g} \cdot \boldsymbol{e}_{\boldsymbol{k}+q k_{d z} \hat{\boldsymbol{z}}, \chi}\right|^{2}\right\rangle_{i s o}=\left|\boldsymbol{\mu}_{e g}\right|^{2} \sum_{q= \pm} \operatorname{Tr}\left[M_{0 q, \beta \alpha}\left(\omega_{\boldsymbol{k}, s}, \boldsymbol{k}\right)\right] \\
& =\frac{4\left|\boldsymbol{\mu}_{e g}\right|^{2}}{3 k_{z d}} \\
& \left\langle\frac{2}{k_{d z}} \sum_{\chi} r_{\boldsymbol{k}-k_{d z} \hat{\boldsymbol{z}}, \chi}\left(\boldsymbol{e}_{\boldsymbol{k}+k_{d z} \hat{\boldsymbol{z}}, \chi} \cdot \boldsymbol{\mu}_{e g}\right)\left(\boldsymbol{\mu}_{g e} \cdot \boldsymbol{e}_{\boldsymbol{k}-k_{d z} \hat{\boldsymbol{z}}, \chi}^{*}\right)\right\rangle_{i s o}=2\left|\boldsymbol{\mu}_{e g}\right|^{2} \operatorname{Tr}\left[M_{r e f, \beta \alpha}^{\chi}\left(\omega_{\boldsymbol{k}, s}, \boldsymbol{k}\right)\right] \\
& =\frac{2\left(r_{\boldsymbol{k}-k_{d z} \hat{\boldsymbol{z}}, s}-r_{\boldsymbol{k}-k_{d z} \hat{\boldsymbol{z}}, p} \frac{k_{d z}^{2}-|\boldsymbol{k}|^{2}}{k_{d z}^{2}+|\boldsymbol{k}|^{2}}\right)\left|\boldsymbol{\mu}_{e g}\right|^{2}}{3 k_{z d}} .
\end{aligned}
$$

Plugging these expressions into Eqs. (S23)- S19b), we obtain Eqs. 5 - 5 . The evaluation of the associated integrals is analytically tractable,

$$
\begin{aligned}
\int_{z_{0}}^{z_{f}} d z J_{\boldsymbol{k}}(z) e^{-i q k_{d z} z} & =\int_{z_{0}}^{z_{f}} d z \boldsymbol{\mu}_{e g} \cdot \boldsymbol{E}_{\boldsymbol{k}} \sqrt{\frac{\hbar \omega_{\boldsymbol{k}}^{S P}}{2 \epsilon_{0} L_{\boldsymbol{k}}}} e^{-\left(\alpha_{\boldsymbol{k} d}+i q k_{d z}\right) z} \\
& =\boldsymbol{\mu}_{e g} \cdot \boldsymbol{E}_{\boldsymbol{k}} \sqrt{\frac{\hbar \omega_{\boldsymbol{k}}^{S P}}{2 \epsilon_{0} L_{\boldsymbol{k}}}}\left(\frac{e^{-\left(\alpha_{\boldsymbol{k} d}+i q k_{z 1}\right) z_{0}-e^{-\left(\alpha_{\boldsymbol{k} d}+i q k_{z 1}\right) z_{f}}}}{\alpha_{\boldsymbol{k} d}+i q k_{d z}}\right) \\
\int_{z_{0}}^{z_{f}} d z\left|J_{\boldsymbol{k}}(z)\right|^{2} & =\int_{z_{0}}^{z_{f}} d z \mid \boldsymbol{\mu}_{e g} \cdot \boldsymbol{E}_{\boldsymbol{k}} \sqrt{\left.\frac{\hbar \omega_{\boldsymbol{k}}^{S P}}{2 \epsilon_{0} L_{\boldsymbol{k}}} e^{-\alpha_{\boldsymbol{k} d} z}\right|^{2}} \\
& =\left|\boldsymbol{\mu}_{e g} \cdot \boldsymbol{E}_{\boldsymbol{k}}\right|^{2} \frac{\hbar \omega_{\boldsymbol{k}}^{S P}}{2 \epsilon_{0} L_{\boldsymbol{k}}}\left(\frac{e^{-2 \alpha_{\boldsymbol{k} d} z_{0}}-e^{-2 \alpha_{\boldsymbol{k} d} z_{f}}}{2 \alpha_{\boldsymbol{k} d}}\right) .
\end{aligned}
$$

where the terms $\boldsymbol{\mu}_{e g} \cdot \boldsymbol{E}_{\boldsymbol{k}} \sqrt{\frac{\hbar \omega_{k}^{S P}}{2 \epsilon_{0} L_{\boldsymbol{k}}}}$ in these expressions are unimportant in Eq. 5 since they cancel out. 

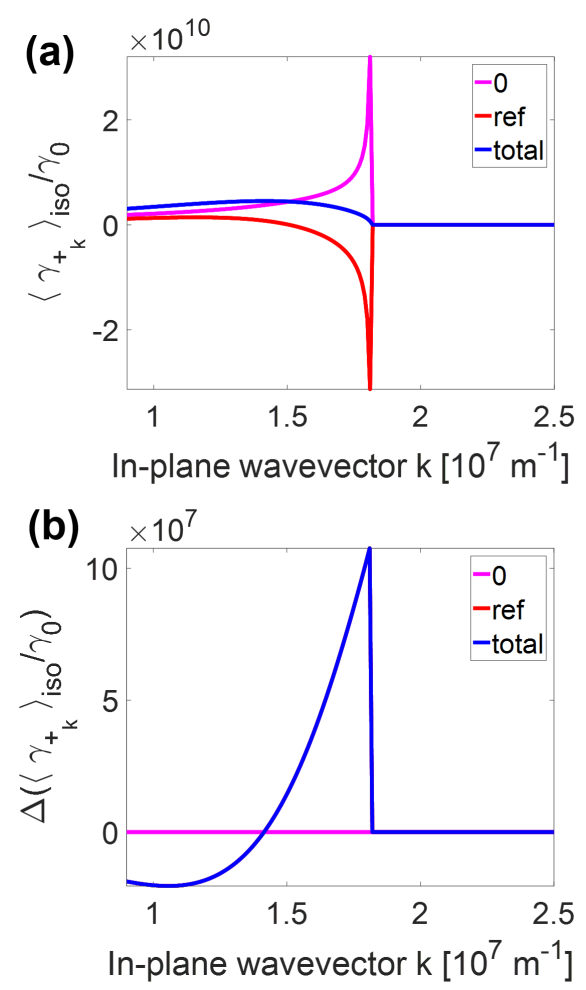

Figura S1. Effects of metal dissipation in plexciton PL rates. (a) PL rates for same conditions as in Fig. $2 \mathrm{p}$, but with lossy metal. No qualitative differences appear when compared to lossless metal case; importantly, van Hove anomalies for $\left\langle\gamma_{+_{\boldsymbol{k}^{*}, i}}\right\rangle_{i_{\text {so }}}$ remain because $\left.\frac{d k_{z d}}{d \omega}\right|_{\omega=\omega_{y_{\boldsymbol{k}^{*}}}}$ still diverges. (b) A close-up on the quantitative PL rate differences $\Delta \frac{\left\langle\gamma_{y_{\boldsymbol{k}}, i}\right\rangle_{i s o}}{\gamma_{S M, 0}}$ with and without metal loss; these differences are negligible when compared to the scale in Fig. $2 p$ or in (a). Note that $\Delta \frac{\left\langle\gamma_{y_{k}, 0}\right\rangle_{\text {iso }}}{\gamma_{S M, 0}}=0$ because it does not depend on the Fresnel coefficient $r_{\boldsymbol{k}-k_{z d} \hat{\boldsymbol{z}}, \chi}$, which contains information about metal loss, so $\Delta \frac{\left\langle\gamma_{y_{\boldsymbol{k}}}\right\rangle_{\text {iso }}}{\gamma_{S M, 0}}=\Delta \frac{\left\langle\gamma_{y_{\boldsymbol{k}}, r e f}\right\rangle_{\text {iso }}}{\gamma_{S M, 0}}$.

\section{EFFECTS OF METAL LOSSES}

In the main text, we have established a condition upon which we expect the superradiant PL rates to be observed experimentally, namely, that the critical wavevector $\boldsymbol{k}^{*}$ is close enough to the anticrossing so the associated plexciton state can be actually considered to arise from strong SP-exciton coupling and the presented polariton theory holds true. In this Section, we consider the robustness of these PL rates against metal losses provided the latter condition is fullfilled.

A complex-valued metal permittivity $\epsilon_{m}(\omega)=\epsilon_{\infty}-\frac{\omega_{P}^{2}}{\omega^{2}+i \omega \gamma}$ for $\gamma \in \Re, \gamma>0$ modifies the modal decomposition of $\hat{\mathcal{E}}_{U H P}$ in Eq. (4). To handle this difficulty, it is convenient to invoke the M-QED formalism due to Dung, Knoll, and Welsch [40] (see also [41, 42]), which bypasses a description of the electromagnetic field in terms of delocalized modes and instead regards it as a polarizable lossy continuum where Eqs. (S27) remains valid. Assuming that the plexciton eigenstates of $H_{\boldsymbol{k}}$ (Eq. (1), still taking $\gamma=0$ ) are good zeroth-order initial states in a Fermi's golden rule PL calculation, it follows that the rate expression in Eq. (S28) still holds, and thus, Eqs. (5) and (6) remain valid. This is a good assumption given that in the strong-coupling regime, the polariton energies are expected to be separated by a Rabi splitting which is much larger than the SP linewidth, $\omega_{+_{k}}-\omega_{-_{k}} \gg \gamma[7$, 20]. The only difference with the previous calculations is that we must account for the fact that metals are not perfect reflectors in the presence of loss, $\left|r_{\boldsymbol{k}-k_{z d} \hat{\boldsymbol{z}}, \chi}\right|^{2} \neq 1$, since $k_{z d}$ becomes complex valued (we need to make sure that $\Im k_{z d}<0$ to guarantee that the modes vanish at $z \rightarrow-\infty$ ). Fig. S1a shows the same plots of Fig. $2 \mathrm{p}$ with the exception that $\gamma=0,07 \mathrm{eV}$ [61]; these figures are qualitatively indistinguishable. Fig. [S1b shows the quantitative difference $\Delta \frac{\left\langle\gamma_{y_{\boldsymbol{k}}, i}\right\rangle_{i s o}}{\gamma_{S M, 0}}=\left.\frac{\left\langle\gamma_{y_{\boldsymbol{k}}, i}\right\rangle_{i s o}}{\gamma_{S M, 0}}\right|_{\gamma=0,07 \mathrm{eV}}-\left.\frac{\left\langle\gamma_{y_{\boldsymbol{k}}, i}\right\rangle_{i s o}}{\gamma_{S M, 0}}\right|_{\gamma=0}$, which is negligible in the scale of each of the normalized PL rates. It is interesting that the van Hove anomalies for $\left\langle\gamma_{y_{k}, i}\right\rangle_{i s o}$ remain even for $\gamma \neq 0$, given that they arise from purely geometric (rather than dynamic) considerations. Thus, we conclude that the predicted PL trends should be robust under a wide range of experimental conditions. 